\title{
UPDATED LIST OF NON-NATIVE ORNAMENTAL PLANTS IN ROMANIA
}

\author{
Mihaela URZICEANU ${ }^{1,2}$, Petronela CAMEN-COMĂNESCU ${ }^{1}$, Eugenia NAGOD $\breve{A}^{1}$, Maria RAICU ${ }^{1}$, Ioana- \\ Minodora SîRBU ${ }^{1,2}$, Paulina ANASTASIU ${ }^{1,2}$ \\ ${ }^{1}$ University of Bucharest, "D. Brandza” Botanic Garden, Cotroceni Șos. 32, RO-060114 Bucharest, Romania \\ ${ }^{2}$ University of Bucharest, Faculty of Biology, Portocalelor Intr. 1-3, RO-060110 Bucharest, Romania \\ email: petronela.comanescu@bio.unibuc.ro
}

\begin{abstract}
The first list of non-native ornamental plants (nNOP) reported as escaped, naturalised or invasive in Romania was published 15 years ago. Since then, new additions to the non-native ornamental flora of Romania have been reported, while others plants have changed their status. As ornamental horticulture is one of the most important pathways for non-native plant introduction worldwide, we evaluated nNOP at the national level and updated the previous list in order to contribute to the development of tools for preventing, detecting, monitoring and managing the spread of nNOP. The updated list includes 264 taxa. Most of these are casual (199 taxa), 37 taxa are naturalised, and 28 taxa are invasive. In addition, the following characteristics were analysed: family, native range, life form, abundance in the country reported as number of localities and temporal trends. Non-native ornamental flora of Romania is dominated by American and Asian taxa, phanerophytes, hemicryptophytes and therophytes. The most widespread species is Robinia pseudoacacia, used not only for ornamental purposes, but also for its economic value. Many taxa (108 taxa) occur only in two to ten localities. $78 \mathrm{nNOP}$ have been reported in the last 20 years. Six taxa are species of European Union concern (Ailanthus altissima, Asclepias syriaca, Cabomba caroliniana, Humulopsis scandens, Impatiens glandulifera, Myriophyllum aquaticum) and require special measures to control and mitigate their populations.
\end{abstract}

Keywords: alien, casual, decorative plants, geographical origin, invasive plants, life form, naturalized, residence time, Romania.

\section{Introduction}

There is strong evidence that ornamental horticulture is a major contributor to plant invasions worldwide $[12,52,25,21]$. Studies conducted in other countries estimate that half of alien naturalized plant species were introduced intentionally, especially being grown for ornamental purposes $[11,29,39]$. There are easy ways to introduce new plants, especially the ecommerce that allows anyone to order plants from various parts of the world $[28,26]$. But once introduced, some of them escape from botanical gardens, public areas or private gardens, become naturalized and compete with native plants $[43,14,25,52,22]$.

It is well known that many characteristics of ornamental plants are responsible for their successful invasiveness [9], and some studies show the most prominent: native range, ease of propagation, a long flowering period, winter hardiness, adaptability to different habitat types, etc. $[41,53,30,52]$. The taxonomic classification of ornamental plants can also be an indicator of their invasivenes [24].

Despite these characteristics, many people buy and cultivate ornamental plants without 
knowing their invasive potential and which species should be avoided for cultivation in different regions [43].

The knowledge of the specific richness and diversity of ornamental alien plants from different regions is a precursor to making predictions about their likely impact [52]. Marking alien ornamental plants in lists is a well-known method for the buying public or stakeholders to be aware of the danger of introducing them into non-native areas and also for any risk assessment [43]. In particular, horticulture professionals and gardeners need to be better informed about the harmfulness of some ornamental plants through available and quality scientific data [23]. Also, using a list of alien ornamental plants together with their characteristics, allows stakeholders to draw up the best monitoring and regulatory measures to reduce their invasion [22]. The discovery that an ornamental plant initially introduced has become invasive over time, also leads to the need to update legislation in order to regulate the import or cultivation conditions [25].

In Romania, Anastasiu et al. [1] published a list of 93 ornamental alien taxa and some of their characteristics, recommending to be careful when alien plants are introduced for ornamental purposes due to the potential of some of them to expand and compete with native species. Fifteen years after the elaboration of this list, the situation in Romania has changed and other new ornamental plants have been listed or reported either in botanical gardens [34, 49, 35], or in urban spaces and private courtyards [47, 4, 48, 33, 8].

Some plants introduced for ornamental and horticultural purposes have caused serious problems in recent years. The best examples are Reynoutria japonica, Echinocystis lobata and Helianthus tuberosus, which escape from cultivation and become abundant, causing severe damage to native vegetation $[15,16]$. None of these were included in the list of Anastasiu et al. [1].

This paper is intended to be an update to the list of alien ornamental plants present in Romanian flora given by Anastasiu et al. [1] and to analyse some characteristics that may play an important role in their invasion. In particular, attributes such as the botanical family, the native range, the life form, the invasive status in Romania, the frequency in Romanian localities and preferred habitat type are presented and discussed.

Because most of these plants are still planted in private gardens and public areas in our country, this list can contribute to the evaluation and development of tools for preventing, detecting, monitoring and managing the spread of non-native ornamental plants, and also for adoption of legal regulations for the horticultural industry with a low risk of plant invasion. Also, this list can be used to raise public awareness and build partnerships with stakeholders.

\section{Material and Methods}

The analyses performed for this paper considered a total of 362 non-native plant species introduced and used for their horticultural or other ornamental value (nNOP) $[5,6,8,20,32,33$, $34,35,46,47,48,49]$. Taxa not reported in the last 50 years were evaluated as vanished and they were excluded from the updated list. The list was completed with taxa reported in the most recent scientific papers dedicated to the flora of botanical gardens $[34,49,35]$, as well as for alien species in general, reported from the national territory [4, 33, 32].

Both, archaeophytes (aliens introduced before 1500 AD) and neophytes [40] were considered in the analyses.

The nomenclature and systematic classification in this study is according to GBIF (Global 
Biodiversity Information Facility, accessed in October and November 2020) [19]. The terminology regarding the invasive status of taxa followed the classification rules recommended by Richardson et al. [44] and Pyšek et al. [40]. The classification for the life forms categories is according to Raunkiær [42].

To reveal the structure of non-native ornamental flora from Romania, the following attributes were analysed: family name, native range, life form, invasive status, number of localities from where taxa were reported, type of habitat, the presence in the previous list of naturalized and invasive ornamental plants [1], the year of the first report of the species on Romanian territory.

To establish from where the reported non-native species came, we considered both continents and geographical regions. This analysis is all the more important as it highlights the most important contributers of non-native species.

Expressing users' preferences for ornamental species, the spectrum of life forms was analysed. In addition, the invasiveness status was highlighted by identifying the species belonging to three categories, depending on the escape capacities, penetration into new territories, reproduction, installation, naturalisation, and invasion of new habitats.

To give an overview on the occurrence of the species at national level and to highlight the most widespread of them, we analysed the distribution of each taxon by the number of localities from where it was mentioned. Comparing the available data, we set a scale starting with species reported from a single locality and ending with the species mentioned in most localities. Moreover, a detailed analysis was performed and thus resulted a scale with seven classes: $\mathbf{1}$ - one locality, $\mathbf{2}$ - two to ten localities, $\mathbf{3}-11$ to 50 localities, $\mathbf{4}-51$ to 100 localities, $\mathbf{5}-101$ to 500 localities, $\mathbf{6}$ - 501 to 1000 localities, 7 - over 1000 localities.

The classification according to the habitats in which the species are present is completed in accordance with the synthesis work of Sîrbu \& Oprea [46].

To illustrate the temporal trend of non-native species over time, we used a time scale based on the years in which each taxon was reported, the starting point being the first reporting in the literature, respectively the year 1779 [46].

Data regarding the status in European Union and the level of impact are according to EASIN Catalogue [18].

\section{Results}

The update list of the non-native plants introduced or used as ornamentals in Romania includes 264 species and subspecies (Table 1). These belong to 80 families. The most important are Asteraceae (33 taxa - 12.5\%), Fabaceae (18 taxa - 6.81\%), Rosaceae (15 taxa - 5.68\%), Solanaceae (10 taxa-3.78\%), and Lamiaceae ( 8 taxa-3.03\%) (Fig. 1). A total of 34 families are represented by only one taxon (12.87\%). 
Table 1: List of non-native ornamental plants in Romania, escaped from cultivation, naturalised and invasive.

\begin{tabular}{|c|c|c|c|c|c|c|c|c|c|c|}
\hline No. & Taxon & Family & Native range & $\begin{array}{l}\text { Life } \\
\text { form }\end{array}$ & $\begin{array}{l}\text { Invasive } \\
\text { status }\end{array}$ & $\begin{array}{l}\text { Classes of } \\
\text { occurrence }\end{array}$ & $\begin{array}{l}\text { Type of } \\
\text { habitat }\end{array}$ & $\begin{array}{l}\text { First } \\
\text { report }\end{array}$ & Status EU & $\begin{array}{l}\text { Is high } \\
\text { impact? }\end{array}$ \\
\hline 1 & Abies nordmanniana Spach & Pinaceae & As (Caucasus) & $\mathrm{Ph}$ & $\mathrm{c}$ & 1 & $\mathrm{a}$ & 1972 & Alien & no \\
\hline 2 & Acer negundo L. & Sapindaceae & $\mathrm{Am} \mathrm{N}$ & $\mathrm{Ph}$ & $\mathrm{i}$ & 5 & $\mathrm{n}, \mathrm{sn}, \mathrm{a}$ & 1946 & Alien & yes \\
\hline 3 & Achillea $\times$ roseoalba Ehrend & Asteraceae & Hybrid & $\mathrm{H}$ & $\mathrm{c}$ & 2 & sn, a & 1987 & Cryptogenic & no \\
\hline 4 & Achillea filipendulina Lam. & Asteraceae & As & $\mathrm{H}$ & $\mathrm{c}$ & 1 & $\mathrm{a}$ & 2017 & Alien & no \\
\hline 5 & Aesculus $\times$ carnea Hayne & Sapindaceae & Hybrid & $\mathrm{Ph}$ & $\mathrm{c}$ & 1 & $\mathrm{a}$ & 2018 & Questionable & no \\
\hline 6 & Aesculus hippocastanum L. & Sapindaceae & $\mathrm{Md}$ & $\mathrm{Ph}$ & $\mathrm{c}$ & 3 & $\mathrm{n}, \mathrm{a}$ & 1866 & Alien & no \\
\hline 7 & $\begin{array}{l}\text { Ailanthus altissima (Mill.) } \\
\text { Swingle }\end{array}$ & Simaroubaceae & As (China) & $\mathrm{Ph}$ & $\mathrm{i}$ & 5 & $\mathrm{n}, \mathrm{sn}, \mathrm{a}$ & 1842 & Alien & yes \\
\hline 8 & $\begin{array}{l}\text { Alangium platanifolium (Siebold } \\
\text { \& Zucc.) Harms }\end{array}$ & Cornaceae & $\begin{array}{l}\text { As (China } \mathrm{C} \text {, } \\
\text { Japan) }\end{array}$ & $\mathrm{Ph}$ & $\mathrm{n}$ & 1 & $\mathrm{a}$ & 2014 & - & - \\
\hline 9 & Albizia julibrissin Durazz. & Fabaceae & As (China) & $\mathrm{Ph}$ & $\mathrm{n}$ & 2 & $\mathrm{a}$ & 1980 & Alien & yes \\
\hline 10 & Alcea rosea L. & Malvaceae & As & $\mathrm{H}$ & $\mathrm{c}$ & 4 & $\mathrm{n}, \mathrm{a}$ & 1816 & Alien & no \\
\hline 11 & Allium subhirsutum L. & Amaryllidaceae & $\mathrm{Md}$ & $\mathrm{G}$ & $\mathrm{c}$ & 1 & $\mathrm{a}$ & 2015 & Alien & no \\
\hline 12 & $\begin{array}{l}\text { Allium tuberosum Rottl. ex } \\
\text { Spreng. }\end{array}$ & Amaryllidaceae & $\begin{array}{l}\text { As (China, } \\
\text { Himalaya) }\end{array}$ & $\mathrm{G}$ & $\mathrm{c}$ & 1 & $\mathrm{a}$ & 2015 & Alien & no \\
\hline 13 & Amaranthus caudatus L. & Amaranthaceae & $\mathrm{Am} \mathrm{S}$ & $\mathrm{T}$ & $\mathrm{n}$ & 3 & $\mathrm{a}$ & 1866 & Alien & no \\
\hline 14 & Amaranthus cruentus L. & Amaranthaceae & Am trop & $\mathrm{T}$ & $\mathrm{n}$ & 3 & $\mathrm{a}$ & 1866 & Alien & no \\
\hline 15 & Amaranthus hypochondriacus L. & Amaranthaceae & $\mathrm{Am} \mathrm{N}$ & $\mathrm{T}$ & $\mathrm{i}$ & 4 & $\mathrm{a}$ & 1866 & Alien & no \\
\hline 16 & Amorpha fruticosa L. & Fabaceae & Am N & $\mathrm{Ph}$ & $\mathrm{i}$ & 5 & $\mathrm{n}, \mathrm{sn}, \mathrm{a}$ & 1898 & Alien & yes \\
\hline 17 & $\begin{array}{l}\text { Anaphalis margaritacea (L.) } \\
\text { Benth. \& Hook.f. }\end{array}$ & Asteraceae & Am N & $\mathrm{H}$ & $\mathrm{c}$ & 2 & $\mathrm{n}, \mathrm{a}$ & 1866 & Alien & no \\
\hline 18 & Antirrhinum majus L. & Plantaginaceae & Md & $\mathrm{T}$ & $\mathrm{c}$ & 3 & $\mathrm{n}, \mathrm{a}$ & 1816 & Alien & no \\
\hline 19 & Aquilegia formosa Fisch. & Ranunculaceae & Am N & $\mathrm{H}$ & $\mathrm{c}$ & 1 & $\mathrm{a}$ & 2015 & - & - \\
\hline 20 & Aquilegia vulgaris L. & Ranunculaceae & Eur W,C,S & $\mathrm{H}$ & $\mathrm{c}$ & 4 & $\mathrm{a}$ & 1816 & Alien & no \\
\hline 21 & Aristolochia macrophylla Lam. & Aristolochiaceae & $\mathrm{Am} \mathrm{N}$ & $\mathrm{Ph}$ & $\mathrm{c}$ & 2 & $\mathrm{a}$ & 1955 & Alien & no \\
\hline
\end{tabular}




\begin{tabular}{|c|c|c|c|c|c|c|c|c|c|c|}
\hline 22 & Asclepias syriaca L. & Apocynaceae & $\mathrm{Am} \mathrm{N}$ & $\mathrm{H}$ & $\mathrm{i}$ & 4 & $\mathrm{n}, \mathrm{sn}, \mathrm{a}$ & 1866 & Alien & yes \\
\hline 23 & Aubrieta parviflora Boiss. & Brassicaceae & $\begin{array}{l}\text { As (Turkey, } \\
\text { Iran) }\end{array}$ & $\mathrm{H}$ & $\mathrm{c}$ & 1 & $\mathrm{a}$ & 2015 & - & - \\
\hline 24 & Bassia scoparia (L.) A.J.Scott & Amaranthaceae & As & $\mathrm{T}$ & $\mathrm{i}$ & 6 & $\mathrm{a}$ & 1816 & Alien & no \\
\hline 25 & Berberis aquifolium Pursh & Berberidaceae & $\mathrm{Am} \mathrm{N}$ & $\mathrm{Ph}$ & $\mathrm{c}$ & 2 & $\mathrm{a}$ & 1994 & Alien & yes \\
\hline 26 & Berberis julianae C.K. Schneid. & Berberidaceae & As (China) & $\mathrm{Ph}$ & $\mathrm{c}$ & 1 & $\mathrm{a}$ & 2015 & Alien & no \\
\hline 27 & Berberis thunbergii DC. & Berberidaceae & As (Japan) & $\mathrm{Ph}$ & $\mathrm{c}$ & 1 & $\mathrm{a}$ & 2017 & Alien & yes \\
\hline 28 & Borago officinalis L. & Boraginaceae & $\mathrm{Md}$ & $\mathrm{T}$ & $\mathrm{c}$ & 3 & $\mathrm{a}$ & 1858 & Alien & no \\
\hline 29 & $\begin{array}{l}\text { Bothriochloa bladhii (Retz.) } \\
\text { S.T.Blake }\end{array}$ & Poaceae & Trop & $\mathrm{H}$ & $\mathrm{n}$ & 2 & $\mathrm{a}$ & 1944 & Alien & no \\
\hline 30 & Brugmansia arborea Steud. & Solanaceae & $\mathrm{Am} \mathrm{S}$ & $\mathrm{Ph}$ & $\mathrm{c}$ & 1 & $\mathrm{a}$ & 2012 & Alien & no \\
\hline 31 & $\begin{array}{l}\text { Brunnera macrophylla } \\
\text { I.M.Johnst. }\end{array}$ & Boraginaceae & As (Caucasus) & $\mathrm{H}$ & $\mathrm{c}$ & 2 & $\mathrm{a}$ & 1947 & Alien & no \\
\hline 32 & Buddleja davidii Franch. & Scrophulariaceae & As E & $\mathrm{Ph}$ & $\mathrm{c}$ & 2 & sn, a & 1961 & Alien & yes \\
\hline 33 & $\begin{array}{l}\text { Bulbine semibarbata (R.Br.) } \\
\text { Haw. }\end{array}$ & Asphodelaceae & Austr & $\mathrm{G}$ & $\mathrm{c}$ & 1 & $\mathrm{a}$ & 2015 & - & - \\
\hline 34 & Buxus sempervirens L. & Buxaceae & $\mathrm{Md}$ & $\mathrm{Ph}$ & $\mathrm{n}$ & 2 & $\mathrm{a}$ & 1958 & Alien & no \\
\hline 35 & Cabomba caroliniana A. Gray & Cabombaceae & $\mathrm{Am} \mathrm{N}, \mathrm{S}$ & $\mathrm{HH}$ & $\mathrm{n}$ & 2 & $\mathrm{n}, \mathrm{sn}$ & 1955 & Alien & yes \\
\hline 36 & Calendula arvensis L. & Asteraceae & Md-Eur C & $\mathrm{T}$ & $\mathrm{c}$ & 3 & $\mathrm{a}$ & 1816 & Alien & no \\
\hline 37 & Calendula officinalis L. & Asteraceae & $\mathrm{Md}$ & $\mathrm{T}$ & $\mathrm{c}$ & 5 & $\mathrm{n}, \mathrm{a}$ & 1779 & Alien & no \\
\hline 38 & Callistephus chinensis Nees & Asteraceae & As E & $\mathrm{T}$ & $\mathrm{c}$ & 3 & $\mathrm{n}, \mathrm{a}$ & 1866 & Alien & no \\
\hline 39 & Campanula medium L. & Campanulaceae & $\mathrm{Md} \mathrm{V}$ & $\mathrm{T}$ & $\mathrm{c}$ & 2 & $\mathrm{a}$ & 1816 & Alien & no \\
\hline 40 & Campsis radicans (L.) Seem. & Bignoniaceae & Am N & $\mathrm{Ph}$ & $\mathrm{c}$ & 2 & $\mathrm{n}, \mathrm{a}$ & 2009 & Alien & no \\
\hline 41 & Caragana arborescens Lam. & Fabaceae & As N & $\mathrm{Ph}$ & $\mathrm{c}$ & 2 & sn, a & 1936 & Alien & no \\
\hline 42 & Carya ovata K.Koch & Juglandaceae & $\mathrm{Am} \mathrm{N}$ & $\mathrm{Ph}$ & $\mathrm{c}$ & 2 & $\mathrm{a}$ & 1960 & Alien & no \\
\hline 43 & Catalpa bignonioides Walter & Bignoniaceae & $\mathrm{Am} \mathrm{N}$ & $\mathrm{Ph}$ & $\mathrm{n}$ & 2 & $\mathrm{a}$ & 1961 & Alien & no \\
\hline 44 & Catalpa fargesii Bur. & Bignoniaceae & As (China) & $\mathrm{Ph}$ & $\mathrm{c}$ & 1 & $\mathrm{a}$ & 2017 & - & - \\
\hline 45 & Catalpa ovata G.Don & Bignoniaceae & As E & $\mathrm{Ph}$ & $\mathrm{c}$ & 1 & $\mathrm{a}$ & 2017 & Alien & no \\
\hline 46 & $\begin{array}{l}\text { Catalpa speciosa Warder ex } \\
\text { Engelm. }\end{array}$ & Bignoniaceae & $\mathrm{Am} \mathrm{N}$ & $\mathrm{Ph}$ & $\mathrm{c}$ & 1 & $\mathrm{a}$ & 2017 & - & - \\
\hline 47 & $\begin{array}{l}\text { Celastrus orbiculatus } \\
\text { C.P.Thunberg ex A.Murray }\end{array}$ & Celastraceae & $\begin{array}{l}\text { As (China, } \\
\text { Japan) }\end{array}$ & $\mathrm{Ph}$ & $\mathrm{c}$ & 1 & $\mathrm{a}$ & 2014 & Alien & yes \\
\hline 48 & Celosia argentea L. & Amaranthaceae & Trop & $\mathrm{T}$ & $\mathrm{c}$ & 2 & $\mathrm{a}$ & 1952 & Alien & no \\
\hline 49 & Celtis occidentalis L. & Cannabaceae & $\mathrm{Am} \mathrm{N}$ & $\mathrm{Ph}$ & $\mathrm{c}$ & 3 & $\mathrm{n}, \mathrm{sn}, \mathrm{a}$ & 1943 & Alien & no \\
\hline
\end{tabular}




\begin{tabular}{|c|c|c|c|c|c|c|c|c|c|c|}
\hline 50 & Cenchrus alopecuroides Thunb. & Poaceae & As E -Austr W & $\mathrm{H}$ & $\mathrm{c}$ & 1 & $\mathrm{a}$ & 2017 & - & - \\
\hline 51 & $\begin{array}{l}\text { Cephalotaxus harringtonii } \\
\text { (Knight ex J.Forbes) K.Koch } \\
\text { var. harringtonii }\end{array}$ & Cephalotaxaceae & As E & $\mathrm{Ph}$ & $\mathrm{c}$ & 1 & $\mathrm{a}$ & 1958 & Alien & no \\
\hline 52 & Cerastium tomentosum L. & Caryophyllaceae & Eur $\mathrm{S}$ & $\mathrm{Ch}$ & $\mathrm{c}$ & 1 & $\mathrm{a}$ & 2015 & Alien & no \\
\hline 53 & $\begin{array}{l}\text { Ceratopteris thalictroides (L.) } \\
\text { Brongn. }\end{array}$ & Pteridaceae & Trop & $\mathrm{HH}$ & $\mathrm{n}$ & 1 & $\mathrm{n}, \mathrm{a}$ & 1974 & Alien & no \\
\hline 54 & Cercis canadensis L. & Fabaceae & $\mathrm{Am} \mathrm{N}$ & $\mathrm{Ph}$ & $\mathrm{c}$ & 1 & $\mathrm{a}$ & 2014 & - & - \\
\hline 55 & Cercis griffithii Boiss. & Fabaceae & As (Afganistan) & $\mathrm{Ph}$ & $\mathrm{c}$ & 1 & $\mathrm{a}$ & 2014 & - & - \\
\hline 56 & Cercis siliquastrum L. & Fabaceae & $\mathrm{Md}$ & $\mathrm{Ph}$ & $\mathrm{c}$ & 2 & $\mathrm{a}$ & 1934 & Alien & no \\
\hline 57 & $\begin{array}{l}\text { Chaenomeles japonica (Thunb.) } \\
\text { Lindl. ex Spach }\end{array}$ & Rosaceae & As E & $\mathrm{Ph}$ & $\mathrm{c}$ & 2 & $\mathrm{a}$ & 1974 & Alien & no \\
\hline 58 & Chaenomeles speciosa Nakai & Rosaceae & As (China) & $\mathrm{Ph}$ & $\mathrm{c}$ & 2 & $\mathrm{a}$ & 2015 & Alien & no \\
\hline 59 & Cheiranthus cheiri L. & Brassicaceae & $\mathrm{Md}$ & $\mathrm{T}$ & $\mathrm{c}$ & 2 & $\mathrm{n}, \mathrm{a}$ & 1954 & Questionable & no \\
\hline 60 & Chrysanthemum indicum L. & Asteraceae & As (China) & $\mathrm{H}$ & $\mathrm{c}$ & 1 & $\mathrm{a}$ & 2011 & Alien & no \\
\hline 61 & Citrus trifoliata L. & Rutaceae & As & $\mathrm{Ph}$ & $\mathrm{c}$ & 1 & $\mathrm{a}$ & 2014 & Alien & no \\
\hline 62 & Cladrastis kentukea Rudd & Fabaceae & $\mathrm{Am} \mathrm{N}$ & $\mathrm{Ph}$ & $\mathrm{c}$ & 1 & $\mathrm{a}$ & 2014 & - & - \\
\hline 63 & Claytonia perfoliata Donn. & Montiaceae & $\mathrm{Am} \mathrm{N}$ & $\mathrm{T}$ & $\mathrm{c}$ & 1 & $\mathrm{a}$ & 2017 & Alien & no \\
\hline 64 & Clematis viticella L. & Ranunculaceae & Eur S, As W & $\mathrm{Ph}$ & $\mathrm{c}$ & 2 & $\mathrm{a}$ & 1880 & Alien & no \\
\hline 65 & Commelina communis L. & Commelinaceae & As temp & $\mathrm{H}$ & $\mathrm{n}$ & 3 & $\mathrm{n}, \mathrm{sn}, \mathrm{a}$ & 1947 & Alien & no \\
\hline 66 & Consolida ajacis (L.) Schur & Ranunculaceae & $\mathrm{Md}$ & $\mathrm{T}$ & $n$ & 3 & $\mathrm{a}$ & 1866 & Alien & no \\
\hline 67 & Coreopsis lanceolata L. & Asteraceae & $\mathrm{Am} \mathrm{N}$ & $\mathrm{H}$ & $\mathrm{c}$ & 2 & $\mathrm{n}, \mathrm{a}$ & 1969 & Alien & no \\
\hline 68 & Coreopsis tinctoria Nutt. & Asteraceae & $\mathrm{Am} \mathrm{N}$ & $\mathrm{T}$ & $\mathrm{c}$ & 3 & $\mathrm{n}, \mathrm{a}$ & 1866 & Alien & no \\
\hline 69 & Cosmos bipinnatus Cav. & Asteraceae & $\mathrm{Am} \mathrm{N}$ & $\mathrm{T}$ & $\mathrm{c}$ & 4 & $\mathrm{n}, \mathrm{a}$ & 1937 & Alien & no \\
\hline 70 & Cotoneaster horizontalis Decne. & Rosaceae & As (China V) & $\mathrm{Ph}$ & $\mathrm{c}$ & 1 & $\mathrm{a}$ & 2015 & Alien & no \\
\hline 71 & Cyclamen coum Mill. & Primulaceae & As (Caucasus) & $\mathrm{G}$ & $\mathrm{c}$ & 1 & $\mathrm{a}$ & 2015 & Alien & no \\
\hline 72 & Cyclamen purpurascens Mill. & Primulaceae & Eur $\mathrm{C}$ & $\mathrm{G}$ & $\mathrm{c}$ & 3 & $\mathrm{n}, \mathrm{sn}, \mathrm{a}$ & 1816 & Alien & no \\
\hline 73 & $\begin{array}{l}\text { Cymbalaria muralis P.Gaertner, } \\
\text { B.Meyer \& Scherb. }\end{array}$ & Plantaginaceae & Eur $\mathrm{S}$ & $\mathrm{H}$ & $\mathrm{n}$ & 3 & $\mathrm{n}, \mathrm{sn}, \mathrm{a}$ & 1816 & Alien & no \\
\hline 74 & Cynara scolymus L. & Asteraceae & $\mathrm{Md}$ & $\mathrm{H}$ & $\mathrm{c}$ & 2 & $\mathrm{a}$ & 2000 & Alien & no \\
\hline 75 & Cytisus scoparius (L.) Link & Fabaceae & Eur W,S\&C & $\mathrm{Ph}$ & $\mathrm{n}$ & 4 & $\mathrm{n}, \mathrm{sn}, \mathrm{a}$ & 1816 & - & - \\
\hline 76 & Datura innoxia Mill. & Solanaceae & Am S,C & $\mathrm{T}$ & $\mathrm{c}$ & 3 & $\mathrm{n}, \mathrm{sn}, \mathrm{a}$ & 1992 & Alien & no \\
\hline 77 & Datura wrightii Regel & Solanaceae & $\mathrm{Am} \mathrm{N}$ & $\mathrm{T}$ & $\mathrm{c}$ & 2 & $\mathrm{a}$ & 2011 & Alien & no \\
\hline
\end{tabular}




\begin{tabular}{|c|c|c|c|c|c|c|c|c|c|c|}
\hline 78 & $\begin{array}{l}\text { Desmodium stenophyllum } \\
\text { Pamp. }\end{array}$ & Fabaceae & $\begin{array}{l}\text { As (Himalaya, } \\
\text { China) }\end{array}$ & $\mathrm{Ph}$ & $\mathrm{n}$ & 1 & $\mathrm{a}$ & 2014 & - & - \\
\hline 79 & Digitalis lutea L. & Plantaginaceae & Eur & $\mathrm{H}$ & $\mathrm{c}$ & 1 & $\mathrm{a}$ & 2015 & Alien & no \\
\hline 80 & $\begin{array}{l}\text { Echinocystis lobata Torr. \& } \\
\text { A.Gray }\end{array}$ & Cucurbitaceae & $\mathrm{Am} \mathrm{N}$ & $\mathrm{T}$ & $\mathrm{i}$ & 6 & $\mathrm{n}, \mathrm{sn}, \mathrm{a}$ & 1904 & Alien & yes \\
\hline 81 & Elaeagnus angustifolia L. & Elaeagnaceae & As & $\mathrm{Ph}$ & $\mathrm{i}$ & 5 & $\mathrm{n}, \mathrm{sn}, \mathrm{a}$ & 1816 & Alien & yes \\
\hline 82 & Eranthis hyemalis Salisb. & Ranunculaceae & $\mathrm{Md}$ & $\mathrm{H}$ & $\mathrm{n}$ & 2 & $\mathrm{n}, \mathrm{sn}, \mathrm{a}$ & 1780 & Alien & no \\
\hline 83 & $\begin{array}{l}\text { Erythranthe guttata (DC.) } \\
\text { G.L.Nesom }\end{array}$ & Phrymaceae & $\mathrm{Am} \mathrm{N}$ & $\mathrm{H}$ & $\mathrm{c}$ & 2 & $\mathrm{a}$ & 1898 & Alien & yes \\
\hline 84 & $\begin{array}{l}\text { Erythranthe moschata (Douglas } \\
\text { ex Lindl.) G.L.Nesom }\end{array}$ & Phrymaceae & $\mathrm{Am} \mathrm{N}$ & $\mathrm{H}$ & $\mathrm{c}$ & 2 & $\mathrm{a}$ & 1871 & Alien & no \\
\hline 85 & Eschscholzia californica Cham. & Papaveraceae & $\mathrm{Am} \mathrm{N}$ & $\mathrm{T}-\mathrm{H}$ & $\mathrm{c}$ & 2 & $\mathrm{a}$ & 1961 & Alien & no \\
\hline 86 & $\begin{array}{l}\text { Euonymus fortunei (Turcz.) } \\
\text { Hand.-Mazz. }\end{array}$ & Celastraceae & As (China) & $\mathrm{Ph}$ & $\mathrm{c}$ & 1 & $\mathrm{a}$ & 2015 & Alien & no \\
\hline 87 & Euphorbia lathyris L. & Euphorbiaceae & $\mathrm{Md}$ & $\mathrm{TH}$ & $\mathrm{c}$ & 3 & $\mathrm{a}$ & 1816 & Alien & no \\
\hline 88 & Euphorbia marginata Pursh & Euphorbiaceae & $\mathrm{Am} \mathrm{N}$ & $\mathrm{T}$ & $\mathrm{n}$ & 3 & $\mathrm{n}, \mathrm{a}$ & 1947 & Alien & no \\
\hline 89 & Euthamia graminifolia (L.) Nutt. & Asteraceae & $\mathrm{Am} \mathrm{N}$ & $\mathrm{H}$ & $\mathrm{n}$ & 2 & $\mathrm{n}, \mathrm{a}$ & 1968 & Alien & no \\
\hline 90 & $\begin{array}{l}\text { Fallopia baldschuanica (Regel) } \\
\text { Holub }\end{array}$ & Polygonaceae & As $\mathrm{E}$ & $\mathrm{Ph}$ & $\mathrm{c}$ & 3 & $\mathrm{n}, \mathrm{a}$ & 1958 & Alien & no \\
\hline 91 & Forsythia suspensa Vahl & Oleaceae & AS (China) & $\mathrm{Ph}$ & $\mathrm{c}$ & 1 & $\mathrm{a}$ & 2015 & Alien & no \\
\hline 92 & Fraxinus americana L. & Oleaceae & $\mathrm{Am} \mathrm{N}$ & $\mathrm{Ph}$ & $\mathrm{c}$ & 3 & $\mathrm{n}, \mathrm{sn}, \mathrm{a}$ & 2008 & Alien & no \\
\hline 93 & Fraxinus pennsylvanica Marshall & Oleaceae & $\mathrm{Am} \mathrm{N}$ & $\mathrm{Ph}$ & $\mathrm{i}$ & 5 & $\mathrm{n}, \mathrm{sn}, \mathrm{a}$ & 1947 & Alien & no \\
\hline 94 & Gaillardia pulchella Foug. & Asteraceae & $\mathrm{Am} \mathrm{N}$ & $\mathrm{T}$ & $\mathrm{c}$ & 2 & $\mathrm{a}$ & 2009 & Alien & no \\
\hline 95 & Gazania rigens (L.) Gaertner & Asteraceae & Afr S & $\mathrm{H}$ & $\mathrm{c}$ & 1 & $\mathrm{a}$ & 2015 & - & - \\
\hline 96 & Ginkgo biloba L. & Ginkgoaceae & As E & $\mathrm{Ph}$ & $\mathrm{c}$ & 2 & $\mathrm{a}$ & 1947 & Alien & no \\
\hline 97 & Gleditsia triacanthos L. & Fabaceae & $\mathrm{Am} \mathrm{N}$ & $\mathrm{Ph}$ & $\mathrm{n}$ & 5 & $\mathrm{n}, \mathrm{sn}, \mathrm{a}$ & 1898 & Alien & yes \\
\hline 98 & Gomphrena globosa L. & Amaranthaceae & Am C,S & $\mathrm{T}$ & $\mathrm{c}$ & 2 & $\mathrm{a}$ & 2014 & Alien & no \\
\hline 99 & Gypsophila altissima L. & Caryophyllaceae & As, Eur cont & $\mathrm{H}$ & $\mathrm{c}$ & 1 & $\mathrm{a}$ & 2015 & Alien & no \\
\hline 100 & Helianthus decapetalus L. & Asteraceae & $\mathrm{Am} \mathrm{N}$ & $\mathrm{H}$ & $\mathrm{n}$ & 4 & $\mathrm{n}, \mathrm{sn}, \mathrm{a}$ & 1948 & Alien & no \\
\hline 101 & Helianthus tuberosus L. & Asteraceae & $\mathrm{Am} \mathrm{N}$ & $\mathrm{H}$ & $\mathrm{i}$ & 5 & $\mathrm{n}, \mathrm{sn}, \mathrm{a}$ & 1858 & Alien & yes \\
\hline 102 & Hemerocallis fulva L. & Asphodelaceae & As E & $\mathrm{H}$ & $\mathrm{c}$ & 4 & $\mathrm{n}, \mathrm{sn}, \mathrm{a}$ & 1816 & Alien & no \\
\hline 103 & Hemerocallis lilioasphodelus L. & Asphodelaceae & As & $\mathrm{H}$ & $\mathrm{c}$ & 3 & $\mathrm{n}, \mathrm{sn}, \mathrm{a}$ & 1816 & Alien & no \\
\hline 104 & Hibiscus moscheutos L. & Malvaceae & $\mathrm{Am} \mathrm{N}$ & $\mathrm{H}$ & $\mathrm{c}$ & 1 & $\mathrm{a}$ & 2011 & Alien & no \\
\hline 105 & Hibiscus syriacus L. & Malvaceae & As E,S & $\mathrm{Ph}$ & $\mathrm{c}$ & 2 & $\mathrm{a}$ & 2004 & Alien & no \\
\hline 106 & Hosta plantaginea Asch. & Asparagaceae & As (China) & $\mathrm{G}$ & $\mathrm{c}$ & 1 & $\mathrm{a}$ & 2017 & Alien & no \\
\hline
\end{tabular}




\begin{tabular}{|c|c|c|c|c|c|c|c|c|c|c|}
\hline 107 & $\begin{array}{l}\text { Humulopsis scandens (Lour.) } \\
\text { Grudz }\end{array}$ & Cannabaceae & As E & $\mathrm{T}$ & $\mathrm{i}$ & 2 & $\mathrm{n}, \mathrm{sn}, \mathrm{a}$ & 1937 & Alien & yes \\
\hline 108 & $\begin{array}{l}\text { Hylotelephium spectabile } \\
\text { (Boreau) H.Ohba }\end{array}$ & Crassulaceae & As E & $\mathrm{H}$ & $\mathrm{c}$ & 2 & $\mathrm{a}$ & 2008 & Alien & no \\
\hline 109 & Iberis amara L. & Brassicaceae & Eur W\&S & $\mathrm{T}$ & $\mathrm{c}$ & 2 & $\mathrm{n}, \mathrm{a}$ & 1866 & Alien & no \\
\hline 110 & Iberis umbellata L. & Brassicaceae & $\mathrm{Md}$ & $\mathrm{T}-\mathrm{TH}$ & $\mathrm{c}$ & 2 & $\mathrm{n}, \mathrm{a}$ & 1866 & Alien & no \\
\hline 111 & Ilex aquifolium L. & Aquifoliaceae & Eur W\&S & $\mathrm{Ph}$ & $\mathrm{n}$ & 2 & $\mathrm{n}, \mathrm{a}$ & 1890 & Alien & no \\
\hline 112 & Impatiens balfourii Hook.f. & Balsaminaceae & As & $\mathrm{H}$ & $\mathrm{c}$ & 3 & $\mathrm{a}$ & 2006 & Alien & no \\
\hline 113 & Impatiens balsamina L. & Balsaminaceae & As SE & $\mathrm{T}$ & $\mathrm{c}$ & 2 & $\mathrm{a}$ & 2005 & Alien & no \\
\hline 114 & Impatiens glandulifera Royle & Balsaminaceae & As (Himalaya) & $\mathrm{T}$ & $\mathrm{i}$ & 5 & $\mathrm{n}, \mathrm{sn}, \mathrm{a}$ & 1892 & Alien & yes \\
\hline 115 & Ipomoea hederacea Jacq. & Convolvulaceae & Am trop & $\mathrm{T}$ & $\mathrm{c}$ & 2 & $\mathrm{a}$ & 1987 & - & - \\
\hline 116 & Ipomoea purpurea (L.) Roth & Convolvulaceae & Am trop & $\mathrm{T}$ & $\mathrm{c}$ & 4 & $\mathrm{a}$ & 1937 & Alien & no \\
\hline 117 & Ipomoea quamoclit L. & Convolvulaceae & Am trop & $\mathrm{T}$ & $\mathrm{c}$ & 1 & $\mathrm{a}$ & 1996 & - & - \\
\hline 118 & Ipomoea tricolor Cav. & Convolvulaceae & Am trop & $\mathrm{T}$ & $\mathrm{c}$ & 1 & $\mathrm{a}$ & 1997 & Alien & no \\
\hline 119 & Iris albicans Lange & Iridaceae & $\mathrm{Md}$ & $\mathrm{G}$ & $\mathrm{c}$ & 1 & $\mathrm{a}$ & 2005 & Alien & no \\
\hline 120 & Iris germanica L. & Iridaceae & $\mathrm{Md}$ & $\mathrm{G}$ & $\mathrm{c}$ & 3 & $\mathrm{n}, \mathrm{a}$ & 1816 & - & - \\
\hline 121 & Iris pallida Lam. & Iridaceae & Eur $\mathrm{S}$ & $\mathrm{G}$ & $\mathrm{c}$ & 2 & $\mathrm{n}, \mathrm{a}$ & 1866 & Alien & no \\
\hline 122 & Juniperus virginiana L. & Cupressaceae & $\mathrm{Am} \mathrm{N}$ & $\mathrm{Ph}$ & $\mathrm{c}$ & 2 & $\mathrm{n}, \mathrm{a}$ & 2009 & Alien & no \\
\hline 123 & Koelreuteria paniculata Laxm. & Sapindaceae & As E & $\mathrm{Ph}$ & $\mathrm{c}$ & 3 & $\mathrm{n}, \mathrm{a}$ & 1964 & Alien & no \\
\hline 124 & $\begin{array}{l}\text { Lagenaria siceraria (Molina) } \\
\text { Standl. }\end{array}$ & Cucurbitaceae & Afr trop & $\mathrm{T}$ & $\mathrm{c}$ & 1 & $\mathrm{sn}$ & 2011 & Alien & no \\
\hline 125 & Lilium bulbiferum L. & Liliaceae & Eur $\mathrm{C}$ & $\mathrm{G}$ & $\mathrm{c}$ & 3 & $\mathrm{n}, \mathrm{a}$ & 1816 & Alien & no \\
\hline 126 & Limnophila heterophylla Benth. & Plantaginaceae & As E & $\mathrm{H}$ & $\mathrm{c}$ & 1 & $\mathrm{a}$ & 1959 & - & - \\
\hline 127 & Lobularia maritima (L.) Desv. & Brassicaceae & $\mathrm{Md}$ & $\mathrm{H}$ & $\mathrm{c}$ & 2 & $\mathrm{a}$ & 1971 & Alien & no \\
\hline 128 & Lolium multiflorum Lam. & Poaceae & $\mathrm{Md}$ & $\mathrm{T}-\mathrm{H}$ & $\mathrm{n}$ & 4 & $\mathrm{n}, \mathrm{sn}, \mathrm{a}$ & 1866 & Alien & yes \\
\hline 129 & Lonicera japonica Thunb. & Caprifoliaceae & As E & $\mathrm{Ph}$ & $\mathrm{c}$ & 2 & $\mathrm{n}, \mathrm{sn}, \mathrm{a}$ & 2010 & Alien & yes \\
\hline 130 & Lonicera periclymenum L. & Caprifoliaceae & $\mathrm{Md}$ & $\mathrm{Ph}$ & $\mathrm{c}$ & 3 & $\mathrm{a}$ & 1816 & Alien & no \\
\hline 131 & Lonicera tatarica L. & Caprifoliaceae & As, Eur E & $\mathrm{Ph}$ & $\mathrm{c}$ & 2 & $\mathrm{a}$ & 2005 & Alien & no \\
\hline 132 & Lupinus albus L. & Fabaceae & As W, Eur SW & $\mathrm{T}$ & $\mathrm{c}$ & 2 & $\mathrm{a}$ & 1947 & Alien & no \\
\hline 133 & Lupinus polyphyllus Lindl. & Fabaceae & $\mathrm{Am} \mathrm{N}$ & $\mathrm{H}$ & $\mathrm{c}$ & 2 & $\mathrm{n}, \mathrm{a}$ & 1947 & Alien & yes \\
\hline 134 & Lycium barbarum L. & Solanaceae & As E & $\mathrm{Ph}$ & $\mathrm{i}$ & 6 & sn, a & 1842 & Alien & no \\
\hline 135 & Macleaya cordata R.Br. & Papaveraceae & As E & $\mathrm{H}$ & $\mathrm{c}$ & 2 & $\mathrm{a}$ & 1947 & Alien & no \\
\hline 136 & Maclura pomifera (Raf)C.K.Schneid. & Moraceae & $\mathrm{Am} \mathrm{N}$ & $\mathrm{Ph}$ & $\mathrm{c}$ & 2 & $\mathrm{n}, \mathrm{a}$ & 1947 & Alien & no \\
\hline
\end{tabular}




\begin{tabular}{|c|c|c|c|c|c|c|c|c|c|c|}
\hline 137 & Malva moschata L. & Malvaceae & $\mathrm{Md}$ & $\mathrm{H}$ & $\mathrm{c}$ & 3 & $\mathrm{n}, \mathrm{sn}$ & 1863 & Alien & no \\
\hline 138 & Malva trimestris Salisb. & Malvaceae & $\mathrm{Md}$ & $\mathrm{T}$ & $\mathrm{c}$ & 2 & $\mathrm{a}$ & 1866 & Alien & no \\
\hline 139 & Malva verticillata L. & Malvaceae & As E & $\mathrm{T}$ & $\mathrm{c}$ & 3 & $\mathrm{a}$ & 1903 & Alien & no \\
\hline 140 & $\begin{array}{l}\text { Matthiola longipetala (Vent.) } \\
\text { DC. subsp. longipetala }\end{array}$ & Brassicaceae & $\mathrm{Md} \mathrm{E}$ & $\mathrm{T}$ & $\mathrm{c}$ & 2 & $\mathrm{a}$ & 1992 & Alien & no \\
\hline 141 & Mirabilis jalapa L. & Nyctagynaceae & $\mathrm{Am} \mathrm{S}$ & $\mathrm{H}$ & $\mathrm{c}$ & 3 & $\mathrm{a}$ & 1937 & Alien & no \\
\hline 142 & $\begin{array}{l}\text { Mirabilis nyctaginea (Michx.) } \\
\text { Mac Mill. }\end{array}$ & Nyctagynaceae & $\mathrm{Am} \mathrm{N}$ & $\mathrm{H}$ & $\mathrm{n}$ & 3 & $\mathrm{a}$ & 1947 & Alien & no \\
\hline 143 & $\begin{array}{l}\text { Monochoria korsakowii Regel \& } \\
\text { Maack }\end{array}$ & Pontederiaceae & As temp & $\mathrm{HH}$ & $\mathrm{c}$ & 2 & $\mathrm{n}, \mathrm{sn}, \mathrm{a}$ & 1989 & Alien & no \\
\hline 144 & Morus alba L. & Moraceae & As E & $\mathrm{Ph}$ & $\mathrm{i}$ & 5 & $\mathrm{n}, \mathrm{sn}, \mathrm{a}$ & 1842 & Alien & yes \\
\hline 145 & Morus nigra L. & Moraceae & As SV & $\mathrm{Ph}$ & $\mathrm{n}$ & 3 & $\mathrm{n}, \mathrm{sn}, \mathrm{a}$ & 1842 & Alien & no \\
\hline 146 & $\begin{array}{l}\text { Myriophyllum aquaticum (Vell.) } \\
\text { Verdc. }\end{array}$ & Haloragaceae & $\mathrm{Am} \mathrm{S}$ & $\mathrm{HH}$ & $\mathrm{c}$ & 1 & $\mathrm{n}, \mathrm{a}$ & 1959 & Alien & yes \\
\hline 147 & $\begin{array}{l}\text { Narcissus poeticus L. subsp. } \\
\text { poeticus }\end{array}$ & Amaryllidaceae & $\mathrm{Md}$ & $\mathrm{G}$ & $\mathrm{n}$ & 3 & sn, a & 1816 & Alien & no \\
\hline 148 & Narcissus pseudonarcissus L. & Amaryllidaceae & Atl-Md & $\mathrm{G}$ & $\mathrm{c}$ & 3 & $\mathrm{a}$ & 1866 & Alien & no \\
\hline 149 & Nelumbo nucifera Gaertn. & Nelumbonaceae & $\begin{array}{l}\text { Eur E, As } \\
\text { subtrop }\end{array}$ & $\mathrm{HH}$ & $\mathrm{n}$ & 2 & $\mathrm{n}, \mathrm{a}$ & 1955 & Alien & no \\
\hline 150 & $\begin{array}{l}\text { Nicandra physalodes (L.) } \\
\text { Gaertn. }\end{array}$ & Solanaceae & $\mathrm{Am} \mathrm{S}$ & $\mathrm{T}$ & $\mathrm{c}$ & 2 & $\mathrm{a}$ & 1858 & Alien & no \\
\hline 151 & Nicotiana alata Link \& Otto & Solanaceae & $\mathrm{Am} \mathrm{S}$ & $\mathrm{T}$ & $\mathrm{c}$ & 3 & $\mathrm{a}$ & 1943 & Alien & no \\
\hline 152 & Nigella damascena L. & Ranunculaceae & Eur $\mathrm{S}$ & $\mathrm{T}$ & $\mathrm{c}$ & 2 & $\mathrm{a}$ & 1816 & Alien & no \\
\hline 153 & $\begin{array}{l}\text { Nymphaea } \times \text { marliacea Hort. } \\
\text { Latour-Marliac }\end{array}$ & Nymphaeaceae & Hybrid & $\mathrm{HH}$ & $\mathrm{c}$ & 1 & $\mathrm{n}, \mathrm{a}$ & 2012 & Questionable & no \\
\hline 154 & Nymphaea lotus L. & Nymphaeaceae & Afr & $\mathrm{HH}$ & $\mathrm{n}$ & 1 & $\mathrm{n}, \mathrm{a}$ & 1798 & Alien & no \\
\hline 155 & Ocimum basilicum L. & Lamiaceae & As & $\mathrm{T}$ & $\mathrm{c}$ & 2 & $\mathrm{a}$ & 1841 & Alien & no \\
\hline 156 & Oenothera biennis L. & Onagraceae & $\mathrm{Am} \mathrm{N}$ & $\mathrm{TH}$ & $\mathrm{i}$ & 5 & $\mathrm{n}, \mathrm{sn}, \mathrm{a}$ & 1816 & Alien & no \\
\hline 157 & $\begin{array}{l}\text { Oenothera gaura W.L.Wagner \& } \\
\text { Hoch }\end{array}$ & Onagraceae & $\mathrm{Am} \mathrm{N}$ & $\mathrm{H}$ & $\mathrm{c}$ & 2 & $\mathrm{a}$ & 1957 & Alien & no \\
\hline 158 & Oenothera glazioviana Micheli & Onagraceae & $\mathrm{Am} \mathrm{N}$ & $\mathrm{TH}$ & $\mathrm{i}$ & 3 & $\mathrm{n}, \mathrm{sn}, \mathrm{a}$ & 1957 & Alien & no \\
\hline 159 & Oenothera odorata Jacq. & Onagraceae & $\mathrm{Am} \mathrm{S}$ & $\mathrm{H}$ & $\mathrm{c}$ & 1 & $\mathrm{a}$ & 1979 & - & - \\
\hline 160 & Oenothera parviflora L. & Onagraceae & $\mathrm{Am} \mathrm{N}$ & $\mathrm{TH}$ & $\mathrm{c}$ & 3 & $\mathrm{n}, \mathrm{sn}, \mathrm{a}$ & 1976 & Alien & no \\
\hline 161 & Oenothera rosea Aiton & Onagraceae & $\mathrm{Am} \mathrm{N}$ & $\mathrm{H}$ & $\mathrm{c}$ & 1 & $\mathrm{a}$ & 2017 & Alien & no \\
\hline 162 & Oxalis rosea Jacq. & Oxalidaceae & $\mathrm{Am} \mathrm{S}$ & G & $\mathrm{c}$ & 2 & $\mathrm{a}$ & 2015 & Alien & no \\
\hline
\end{tabular}




\begin{tabular}{|c|c|c|c|c|c|c|c|c|c|c|}
\hline 163 & $\begin{array}{l}\text { Papaver atlanticum (Ball) } \\
\text { Cosson }\end{array}$ & Papaveraceae & Afr & $\mathrm{T}, \mathrm{H}$ & $\mathrm{c}$ & 1 & $\mathrm{a}$ & 2015 & Alien & no \\
\hline 164 & Papaver orientale L. & Papaveraceae & As & $\mathrm{H}$ & $\mathrm{c}$ & 1 & $\mathrm{a}$ & 2015 & Alien & no \\
\hline 165 & Papaver somniferum L. & Papaveraceae & As & $\mathrm{T}$ & $\mathrm{c}$ & 3 & $\mathrm{a}$ & 1816 & Alien & no \\
\hline 166 & $\begin{array}{l}\text { Parthenocissus inserta } \\
\text { (A.Kerner) Fritsch }\end{array}$ & Vitaceae & $\mathrm{Am} \mathrm{N}$ & $\mathrm{Ph}$ & $\mathrm{i}$ & 5 & $\mathrm{n}, \mathrm{sn}, \mathrm{a}$ & 1898 & - & - \\
\hline 167 & $\begin{array}{l}\text { Parthenocissus quinquefolia (L.) } \\
\text { Planch. }\end{array}$ & Vitaceae & $\mathrm{Am} \mathrm{N}$ & $\mathrm{Ph}$ & $\mathrm{c}$ & 3 & $\mathrm{n}, \mathrm{sn}, \mathrm{a}$ & 1866 & Alien & no \\
\hline 168 & $\begin{array}{l}\text { Parthenocissus tricuspidata } \\
\text { Planch. }\end{array}$ & Vitaceae & As E & $\mathrm{Ph}$ & $\mathrm{c}$ & 2 & $\mathrm{a}$ & 2008 & Alien & no \\
\hline 169 & Paulownia tomentosa Steud. & Paulowniaceae & As E & $\mathrm{Ph}$ & $\mathrm{c}$ & 2 & $\mathrm{a}$ & 1970 & Alien & yes \\
\hline 170 & Perilla frutescens Britton. & Lamiaceae & As SE & $\mathrm{T}$ & $\mathrm{c}$ & 3 & $\mathrm{n}, \mathrm{sn}, \mathrm{a}$ & 2005 & Alien & no \\
\hline 171 & Persicaria orientalis (L.) Spach & Polygonaceae & As E & $\mathrm{T}$ & $\mathrm{c}$ & 3 & $\mathrm{a}$ & 1866 & Alien & no \\
\hline 172 & $\begin{array}{l}\text { Petrosedum ochroleucum subsp. } \\
\text { ochroleucum }\end{array}$ & Crassulaceae & $\mathrm{Md}$ & $\mathrm{Ch}$ & $\mathrm{c}$ & 1 & $\mathrm{a}$ & 1959 & Alien & no \\
\hline 173 & $\begin{array}{l}\text { Petunia } \times \text { atkinsiana D.Don ex } \\
\text { Loud }\end{array}$ & Solanaceae & Hybrid & $\mathrm{T}$ & $\mathrm{c}$ & 3 & $\mathrm{a}$ & 1959 & Alien & no \\
\hline 174 & $\begin{array}{l}\text { Petunia axillaris (Lam.) Britton, } \\
\text { Sterns \& Poggenb. }\end{array}$ & Solanaceae & $\mathrm{Am} \mathrm{S}$ & $\mathrm{T}$ & $\mathrm{c}$ & 1 & $\mathrm{a}$ & 2017 & Alien & no \\
\hline 175 & $\begin{array}{l}\text { Petunia integrifolia Schinz \& } \\
\text { Thell. }\end{array}$ & Solanaceae & $\mathrm{Am} \mathrm{S}$ & $\mathrm{T}$ & $\mathrm{c}$ & 2 & $\mathrm{a}$ & 1939 & Alien & no \\
\hline 176 & $\begin{array}{l}\text { Phedimus spurius (M.Bieb.) 't } \\
\text { Hart }\end{array}$ & Crassulaceae & As W & $\mathrm{H}$ & $\mathrm{c}$ & 3 & $\mathrm{a}$ & 1871 & Alien & no \\
\hline 177 & Philadelphus pubescens Loisel. & Hydrangeaceae & $\mathrm{Am} \mathrm{N}$ & $\mathrm{Ph}$ & $\mathrm{c}$ & 2 & $\mathrm{a}$ & 2010 & Cryptogenic & no \\
\hline 178 & $\begin{array}{l}\text { Physocarpus opulifolius (L.) } \\
\text { Maxim. }\end{array}$ & Rosaceae & $\mathrm{Am} \mathrm{N}$ & $\mathrm{Ph}$ & $\mathrm{c}$ & 2 & $\mathrm{a}$ & 1844 & Alien & no \\
\hline 179 & Platanus hybrida Brot. & Platanaceae & Hybrid & $\mathrm{Ph}$ & $\mathrm{c}$ & 2 & $\mathrm{n}, \mathrm{a}$ & 1968 & Questionable & no \\
\hline 180 & $\begin{array}{l}\text { Platycladus orientalis (L.) } \\
\text { Franco }\end{array}$ & Cupressaceae & As E & $\mathrm{Ph}$ & $\mathrm{c}$ & 2 & $\mathrm{a}$ & 1960 & - & - \\
\hline 181 & Populus canadensis Moench & Salicaceae & Hybrid & $\mathrm{Ph}$ & $\mathrm{c}$ & 3 & sn, a & 2006 & Alien & no \\
\hline 182 & Portulaca grandiflora Hook. & Portulacaceae & $\mathrm{Am} \mathrm{S}$ & $\mathrm{T}$ & $\mathrm{c}$ & 3 & $\mathrm{a}$ & 1937 & Alien & no \\
\hline 183 & $\begin{array}{l}\text { Potentilla indica (Andrews) } \\
\text { T.Wolf }\end{array}$ & Rosaceae & As S\&E & $\mathrm{H}$ & $\mathrm{n}$ & 2 & $\mathrm{a}$ & 1949 & Alien & no \\
\hline
\end{tabular}




\begin{tabular}{|c|c|c|c|c|c|c|c|c|c|c|}
\hline 184 & Prunus laurocerasus L. & Rosaceae & As & $\mathrm{Ph}$ & $\mathrm{c}$ & 2 & $\mathrm{a}$ & 1987 & Alien & no \\
\hline 185 & Prunus serotina Ehrh. & Rosaceae & $\mathrm{Am} \mathrm{N}$ & $\mathrm{Ph}$ & $\mathrm{i}$ & 4 & $\mathrm{n}, \mathrm{sn}, \mathrm{a}$ & 1995 & Alien & yes \\
\hline 186 & Pseudofumaria lutea (L.) Borkh. & Papaveraceae & Eur $\mathrm{C}$ & $\mathrm{H}$ & $\mathrm{c}$ & 1 & $\mathrm{a}$ & 1999 & Alien & no \\
\hline 187 & $\begin{array}{l}\text { Pseudotsuga menziesii (Mirb.) } \\
\text { Franco }\end{array}$ & Pinaceae & $\mathrm{Am} \mathrm{N}$ & $\mathrm{Ph}$ & $\mathrm{c}$ & 3 & $\mathrm{a}$ & 1900 & Alien & no \\
\hline 188 & Ptelea trifoliata L. & Rutaceae & $\mathrm{Am} \mathrm{N}$ & $\mathrm{Ph}$ & $\mathrm{c}$ & 2 & $\mathrm{a}$ & 1961 & Alien & no \\
\hline 189 & Pteris multifida Poir. & Pteridaceae & As E & $\mathrm{H}$ & $\mathrm{n}$ & 2 & $\mathrm{a}$ & 1974 & Alien & no \\
\hline 190 & Quercus rubra L. & Fagaceae & $\mathrm{Am} \mathrm{N}$ & $\mathrm{Ph}$ & $\mathrm{n}$ & 3 & sn, a & 1962 & Alien & no \\
\hline 191 & Reseda odorata L. & Resedaceae & $\mathrm{Md}$ & $\mathrm{T}$ & $\mathrm{c}$ & 2 & $\mathrm{a}$ & 1866 & Alien & no \\
\hline 192 & $\begin{array}{l}\text { Reynoutria } \times \text { bohemica Chrtek } \\
\& \text { Chrtková }\end{array}$ & Polygonaceae & Hybrid & $\mathrm{H}$ & i & 5 & $\mathrm{n}, \mathrm{sn}, \mathrm{a}$ & 2004 & Alien & no \\
\hline 193 & Reynoutria japonica Houtt. & Polygonaceae & As E & G-H & $\mathrm{i}$ & 5 & $\mathrm{n}, \mathrm{sn}, \mathrm{a}$ & 1940 & Alien & yes \\
\hline 194 & Reynoutria sachalinensis Nakai & Polygonaceae & As E & $\mathrm{H}$ & $\mathrm{n}$ & 3 & $\mathrm{n}, \mathrm{sn}, \mathrm{a}$ & 1947 & Questionable & yes \\
\hline 195 & Rhus typhina L. & Anacardiaceae & $\mathrm{Am} \mathrm{N}$ & $\mathrm{Ph}$ & $\mathrm{n}$ & 4 & $\mathrm{n}, \mathrm{a}$ & 1866 & Alien & yes \\
\hline 196 & Ribes aureum Pursh & Grossulariaceae & $\mathrm{Am} \mathrm{N}$ & $\mathrm{Ph}$ & $\mathrm{c}$ & 2 & $\mathrm{a}$ & 1842 & Alien & no \\
\hline 197 & Ricinus communis L. & Euphorbiaceae & Afr trop & $\mathrm{T}$ & $\mathrm{c}$ & 3 & sn, a & 1947 & Alien & yes \\
\hline 198 & Robinia pseudoacacia L. & Fabaceae & $\mathrm{Am} \mathrm{N}$ & $\mathrm{Ph}$ & $\mathrm{i}$ & 7 & $\mathrm{n}, \mathrm{sn}, \mathrm{a}$ & 1816 & Alien & yes \\
\hline 199 & Robinia viscosa Vent. & Fabaceae & $\mathrm{Am} \mathrm{N}$ & $\mathrm{Ph}$ & $\mathrm{n}$ & 2 & $\mathrm{a}$ & 2005 & Alien & no \\
\hline 200 & Rosa $\times$ alba L. & Rosaceae & Hybrid & $\mathrm{Ph}$ & $\mathrm{c}$ & 2 & $\mathrm{a}$ & 1836 & Alien & no \\
\hline 201 & Rosa foetida Herrm. & Rosaceae & As SW & $\mathrm{Ph}$ & $\mathrm{c}$ & 2 & $\mathrm{a}$ & 1866 & Alien & no \\
\hline 202 & Rosa rugosa Thunb. & Rosaceae & As E & $\mathrm{Ph}$ & $\mathrm{c}$ & 2 & $\mathrm{a}$ & 1995 & Alien & yes \\
\hline 203 & Rubus phoenicolasius Maxim. & Rosaceae & As E & $\mathrm{Ph}$ & $\mathrm{c}$ & 2 & sn, a & 1956 & Alien & no \\
\hline 204 & Rudbeckia fulgida Ait. & Asteraceae & $\mathrm{Am} \mathrm{N}$ & $\mathrm{H}$ & $\mathrm{c}$ & 1 & $\mathrm{a}$ & 2015 & Alien & no \\
\hline 205 & Rudbeckia hirta L. & Asteraceae & $\mathrm{Am} \mathrm{N}$ & $\mathrm{H}$ & $\mathrm{c}$ & 2 & $\mathrm{n}, \mathrm{a}$ & 2008 & Alien & no \\
\hline 206 & Rudbeckia laciniata L. & Asteraceae & $\mathrm{Am} \mathrm{N}$ & $\mathrm{H}$ & $\mathrm{i}$ & 5 & $\mathrm{n}, \mathrm{sn}, \mathrm{a}$ & 1855 & Alien & no \\
\hline 207 & Rudbeckia triloba L. & Asteraceae & $\mathrm{Am} \mathrm{N}$ & $\mathrm{H}$ & $\mathrm{c}$ & 2 & sn, a & 2004 & Alien & no \\
\hline 208 & Ruta graveolens L. & Rutaceae & $\mathrm{Md}$ & $\mathrm{Ch}$ & $\mathrm{c}$ & 2 & sn, a & 1925 & Alien & no \\
\hline 209 & Sagittaria latifolia Willd. & Alismataceae & $\mathrm{Am} \mathrm{N}$ & $\mathrm{HH}$ & $\mathrm{n}$ & 2 & $\mathrm{n}, \mathrm{sn}, \mathrm{a}$ & 1966 & Alien & yes \\
\hline 210 & Salix babylonica L. & Salicaceae & As E & $\mathrm{Ph}$ & $\mathrm{c}$ & 2 & $\mathrm{a}$ & 1842 & Alien & no \\
\hline 211 & Salvia desoleana Atzei \& V.Picci & Lamiaceae & Md (Sardinia) & $\mathrm{Ch}$ & $\mathrm{c}$ & 1 & $\mathrm{a}$ & 2015 & - & - \\
\hline 212 & Salvia sclarea L. & Lamiaceae & $\mathrm{Md}$ & $\mathrm{H}$ & $\mathrm{c}$ & 3 & $\mathrm{a}$ & 1816 & Alien & no \\
\hline 213 & Salvia splendens Sellow ex Nees & Lamiaceae & $\mathrm{Am} \mathrm{S}$ & $\mathrm{T}$ & $\mathrm{c}$ & 1 & $\mathrm{a}$ & 2005 & Alien & no \\
\hline 214 & Salvia tiliifolia Vahl & Lamiaceae & $\mathrm{Am} \mathrm{C}$ & $\mathrm{T}$ & $\mathrm{c}$ & 1 & $\mathrm{a}$ & 2015 & - & - \\
\hline 215 & Salvia verbenaca L. & Lamiaceae & Atl-Md & $\mathrm{H}$ & $\mathrm{c}$ & 2 & $\mathrm{n}, \mathrm{sn}, \mathrm{a}$ & 1969 & Alien & no \\
\hline
\end{tabular}




\begin{tabular}{|c|c|c|c|c|c|c|c|c|c|c|}
\hline 216 & Sassafras albidum (Nutt.) Nees & Lauraceae & $\mathrm{Am} \mathrm{N}$ & $\mathrm{Ph}$ & $\mathrm{c}$ & 1 & $\mathrm{a}$ & 2014 & - & - \\
\hline 217 & Scilla siberica Andrews & Asparagaceae & $\begin{array}{l}\text { As W, Eur E } \\
\text { (Russia) }\end{array}$ & $\mathrm{G}$ & $\mathrm{c}$ & 2 & sn, a & 1939 & Alien & no \\
\hline 218 & Securigera cretica (L.) Lassen & Fabaceae & $\mathrm{Md}$ & $\mathrm{T}$ & $\mathrm{c}$ & 2 & $\mathrm{a}$ & 1842 & - & - \\
\hline 219 & Sedum dasyphyllum L. & Crassulaceae & $\mathrm{Md}$ & $\mathrm{H}$ & $\mathrm{c}$ & 2 & $\mathrm{n}, \mathrm{a}$ & 1816 & Alien & no \\
\hline 220 & Sedum sarmentosum Bunge & Crassulaceae & As E & $\mathrm{H}$ & c & 3 & $\mathrm{a}$ & 2011 & Alien & no \\
\hline 221 & Sempervivum tectorum L. & Crassulaceae & Eur C,W,S & $\mathrm{H}$ & $\mathrm{c}$ & 3 & $\mathrm{n}, \mathrm{a}$ & 1816 & Alien & no \\
\hline 222 & Sicyos angulatus L. & Cucurbitaceae & $\mathrm{Am} \mathrm{N}$ & $\mathrm{T}$ & i & 3 & $\mathrm{n}, \mathrm{sn}, \mathrm{a}$ & 1816 & Alien & yes \\
\hline 223 & $\begin{array}{l}\text { Silene chalcedonica } \\
\text { E.H.L.Krause }\end{array}$ & Caryophyllaceae & As W, Eur E & $\mathrm{H}$ & $\mathrm{c}$ & 2 & $\mathrm{a}$ & 1866 & Alien & no \\
\hline 224 & Silene pendula L. & Caryophyllaceae & $\mathrm{Md}$ & $\mathrm{T}$ & $\mathrm{c}$ & 2 & $\mathrm{a}$ & 1877 & Alien & no \\
\hline 225 & Silene sibirica Pers. & Caryophyllaceae & As (Siberia) & $\mathrm{H}$ & $\mathrm{c}$ & 2 & $\mathrm{a}$ & 1960 & Alien & no \\
\hline 226 & Silphium perfoliatum L. & Asteraceae & $\mathrm{Am} \mathrm{N}$ & $\mathrm{H}$ & $\mathrm{c}$ & 1 & $\mathrm{a}$ & 1994 & Alien & no \\
\hline 227 & Sisyrinchium montanum Greene & Iridaceae & $\mathrm{Am} \mathrm{N}$ & $\mathrm{H}$ & i & 4 & $\mathrm{n}, \mathrm{sn}, \mathrm{a}$ & 1934 & Alien & no \\
\hline 228 & Smilax excelsa L. & Smilacaceae & As (Caucasus) & $\mathrm{H}$ & $\mathrm{n}$ & 1 & $\mathrm{a}$ & 2014 & Alien & no \\
\hline 229 & Solanum citrullifolium A.Braun & Solanaceae & $\mathrm{Am} \mathrm{C}$ & $\mathrm{T}$ & $\mathrm{c}$ & 2 & $\mathrm{a}$ & 1941 & Alien & no \\
\hline 230 & Solidago canadensis L. & Asteraceae & $\mathrm{Am} \mathrm{N}$ & $\mathrm{H}$ & $\mathrm{i}$ & 5 & $\mathrm{n}, \mathrm{sn}, \mathrm{a}$ & 1866 & Alien & yes \\
\hline 231 & Solidago gigantea Aiton & Asteraceae & $\mathrm{Am} \mathrm{N}$ & $\mathrm{H}$ & i & 5 & $\mathrm{n}, \mathrm{sn}, \mathrm{a}$ & 1886 & Alien & yes \\
\hline 232 & Sorbaria sorbifolia (L.) A.Braun & Rosaceae & As $\mathrm{N}$ & $\mathrm{Ph}$ & $\mathrm{c}$ & 2 & $\mathrm{a}$ & 2009 & Alien & no \\
\hline 233 & Sorghum bicolor (L.) Moench & Poaceae & Afr & $\mathrm{T}$ & $\mathrm{c}$ & 3 & $\mathrm{n}, \mathrm{a}$ & 1842 & Alien & no \\
\hline 234 & Spartium junceum L. & Fabaceae & $\mathrm{Md}$ & $\mathrm{Ph}$ & $\mathrm{c}$ & 2 & $\mathrm{a}$ & 1866 & Alien & no \\
\hline 235 & Spiraea $\times$ billardii Hérincq & Rosaceae & Hybrid & $\mathrm{Ph}$ & $\mathrm{c}$ & 1 & $\mathrm{a}$ & 2002 & Alien & no \\
\hline 236 & $\begin{array}{l}\text { Spiraea } \times \text { vanhouttei }(\text { Briot }) \\
\text { Zabel }\end{array}$ & Rosaceae & Hybrid & $\mathrm{Ph}$ & $\mathrm{c}$ & 2 & $\mathrm{n}, \mathrm{a}$ & 1971 & Alien & no \\
\hline 237 & Spiraea japonica L.f. & Rosaceae & As E & $\mathrm{Ph}$ & $\mathrm{c}$ & 2 & $\mathrm{n}, \mathrm{a}$ & 1969 & Alien & no \\
\hline 238 & Stachys byzantina K.Koch & Lamiaceae & As SW & $\mathrm{H}$ & $\mathrm{c}$ & 3 & $\mathrm{n}, \mathrm{sn}, \mathrm{a}$ & 1939 & Alien & no \\
\hline 239 & $\begin{array}{l}\text { Styphnolobium japonicum (L.) } \\
\text { Schott }\end{array}$ & Fabaceae & As E & $\mathrm{Ph}$ & $\mathrm{c}$ & 2 & $\bar{a}$ & 1961 & Alien & no \\
\hline 240 & $\begin{array}{l}\text { Symphoricarpos albus (L.) } \\
\text { C.Koch }\end{array}$ & Caprifoliaceae & $\mathrm{Am} \mathrm{N}$ & $\mathrm{Ph}$ & $\mathrm{c}$ & 3 & $\bar{a}$ & 1947 & Alien & no \\
\hline 241 & $\begin{array}{l}\text { Symphyotrichum lanceolatum } \\
\text { (Willd.) G.L.Nesom }\end{array}$ & Asteraceae & $\mathrm{Am} \mathrm{N}$ & $\mathrm{H}$ & $\mathrm{i}$ & 5 & $\mathrm{n}, \mathrm{sn}, \mathrm{a}$ & 1940 & Alien & no \\
\hline 242 & $\begin{array}{l}\text { Symphyotrichum novae-angliae } \\
\text { (L.) G.L.Nesom }\end{array}$ & Asteraceae & $\mathrm{Am} \mathrm{N}$ & $\mathrm{H}$ & $\mathrm{c}$ & 2 & $\mathrm{n}, \mathrm{a}$ & 1939 & Alien & no \\
\hline
\end{tabular}




\begin{tabular}{|c|c|c|c|c|c|c|c|c|c|c|}
\hline 243 & $\begin{array}{l}\text { Symphyotrichum novi-belgii } \\
\text { (L.) G.L.Nesom }\end{array}$ & Asteraceae & $\mathrm{Am} \mathrm{N}$ & $\mathrm{H}$ & $\mathrm{c}$ & 4 & $\mathrm{n}, \mathrm{a}$ & 1945 & Alien & yes \\
\hline 244 & $\begin{array}{l}\text { Symphyotrichum salignum } \\
\text { (Willd.) G. L. Nesom }\end{array}$ & Asteraceae & Hybrid & $\mathrm{H}$ & $\mathrm{i}$ & 3 & $\mathrm{n}, \mathrm{sn}, \mathrm{a}$ & 1780 & Alien & no \\
\hline 245 & $\begin{array}{l}\text { Symphyotrichum tradescantii } \\
\text { (L.) G. L. Nesom }\end{array}$ & Asteraceae & $\mathrm{Am} \mathrm{N}$ & $\mathrm{H}$ & $\mathrm{c}$ & 2 & $\mathrm{n}, \mathrm{a}$ & 1939 & Alien & no \\
\hline 246 & $\begin{array}{l}\text { Symphyotrichum versicolor } \\
\text { (Willd.) G. L. Nesom }\end{array}$ & Asteraceae & Hybrid & $\mathrm{H}$ & $\mathrm{c}$ & 3 & $\mathrm{n}, \mathrm{a}$ & 1959 & Alien & no \\
\hline 247 & Tagetes erecta L. & Asteraceae & $\mathrm{Am} \mathrm{C}$ & $\mathrm{T}$ & $\mathrm{c}$ & 2 & $\mathrm{a}$ & 1961 & Alien & no \\
\hline 248 & Tamarix tetrandra Pall.exM.Bieb. & Tamaricaceae & Eur SE, AsiaSW & $\mathrm{Ph}$ & $\mathrm{c}$ & 2 & $\mathrm{a}$ & 1947 & Alien & yes \\
\hline 249 & Tanacetum balsamita L. & Asteraceae & As SW & $\mathrm{H}$ & $\mathrm{c}$ & 3 & $\mathrm{a}$ & 1841 & Alien & no \\
\hline 250 & Tanacetum parthenium Sch.Bip. & Asteraceae & Eur SE & $\mathrm{H}$ & $\mathrm{c}$ & 5 & $\mathrm{n}, \mathrm{sn}, \mathrm{a}$ & 1816 & Alien & no \\
\hline 251 & Tarenaya spinosa Raf. & Cleomaceae & $\mathrm{Am} \mathrm{S}$ & $\mathrm{T}$ & $\mathrm{c}$ & 1 & $\mathrm{a}$ & 2014 & Alien & no \\
\hline 252 & Taxodium distichum Rich. & Cupressaceae & Am C (Mexic) & $\mathrm{Ph}$ & $\mathrm{n}$ & 2 & $\mathrm{n}, \mathrm{sn}, \mathrm{a}$ & 1958 & Alien & no \\
\hline 253 & Thladiantha dubia Bunge & Cucurbitaceae & As E & $\mathrm{G}$ & $\mathrm{n}$ & 5 & $\mathrm{n}, \mathrm{a}$ & 1925 & Alien & no \\
\hline 254 & Tradescantia fluminensis Vell. & Commelinaceae & $\mathrm{Am} \mathrm{S}$ & $\mathrm{H}$ & $\mathrm{c}$ & 2 & $\mathrm{a}$ & 1999 & Alien & yes \\
\hline 255 & Tradescantia virginiana L. & Commelinaceae & $\mathrm{Am} \mathrm{N}$ & $\mathrm{H}$ & $\mathrm{c}$ & 2 & $\mathrm{a}$ & 1909 & Alien & no \\
\hline 256 & Tropaeolum majus L. & Tropaeolaceae & $\mathrm{Am} \mathrm{S}$ & $\mathrm{T}$ & $\mathrm{c}$ & 2 & $\mathrm{a}$ & 2003 & Alien & no \\
\hline 257 & Tulipa gesneriana L. & Liliaceae & As W, Md E & $\mathrm{G}$ & $\mathrm{c}$ & 2 & $\mathrm{a}$ & 1866 & Alien & no \\
\hline 258 & Ulmus pumila L. & Ulmaceae & As $\mathrm{C}, \mathrm{E}$ & $\mathrm{Ph}$ & $\mathrm{n}$ & 3 & $\mathrm{n}, \mathrm{a}$ & 1968 & Alien & no \\
\hline 259 & $\begin{array}{l}\text { Viburnum rhytidophyllum } \\
\text { Hemsl. ex Forb. \& Hemsl. }\end{array}$ & Adoxaceae & As E & $\mathrm{Ph}$ & $\mathrm{c}$ & 1 & $\mathrm{a}$ & 2004 & Alien & no \\
\hline 260 & Vinca major L. & Apocynaceae & $\mathrm{Md}$ & $\mathrm{H}$ & $\mathrm{c}$ & 2 & $\mathrm{a}$ & 1863 & Alien & no \\
\hline 261 & $\begin{array}{l}\text { Viola } \times \text { wittrockiana Gams ex } \\
\text { Nauenb. \& Buttler }\end{array}$ & Violaceae & Hybrid & $\mathrm{H}$ & $\mathrm{c}$ & 2 & $\mathrm{a}$ & 2009 & Questionable & no \\
\hline 262 & Wisteria sinensis Sweet & Fabaceae & As E & $\mathrm{Ph}$ & $\mathrm{c}$ & 2 & $\mathrm{a}$ & 2005 & Alien & no \\
\hline 263 & Yucca filamentosa L. & Asparagaceae & $\mathrm{Am}$ & $\mathrm{Ph}$ & $\mathrm{c}$ & 2 & $\mathrm{a}$ & 2011 & Alien & no \\
\hline 264 & Zinnia elegans Jacq. & Asteraceae & $\mathrm{Am} \mathrm{C}$ & $\mathrm{T}$ & $\mathrm{c}$ & 2 & $\mathrm{a}$ & 2005 & Alien & no \\
\hline
\end{tabular}

Abbreviations: Native range: Afr - Africa, Am - America, As - Asia, Austr - Australia, Eur - Europe, Euras - Eurasia, Anat - Anatolia, Atl - Atlantic, Temp - Temperate, Trop - Tropical, Md - Mediterranean, N - North, E - East, S - South, W - West, C - Central. Life form: Ch - Chamaephyte, G - Geophyte, H Hemicryptophyte, HH - Helohydatophyte, Ph - Phanerophyte, T - Therophyte, TH - Hemitherophytes. Status of invasiveness in Romania: c - casual, $\mathrm{n}-$ naturalized, i - invasive. Type of habitat: $\mathrm{n}$ - natural, sn - semi-natural, a - artificial. Others: EU - European Union. 


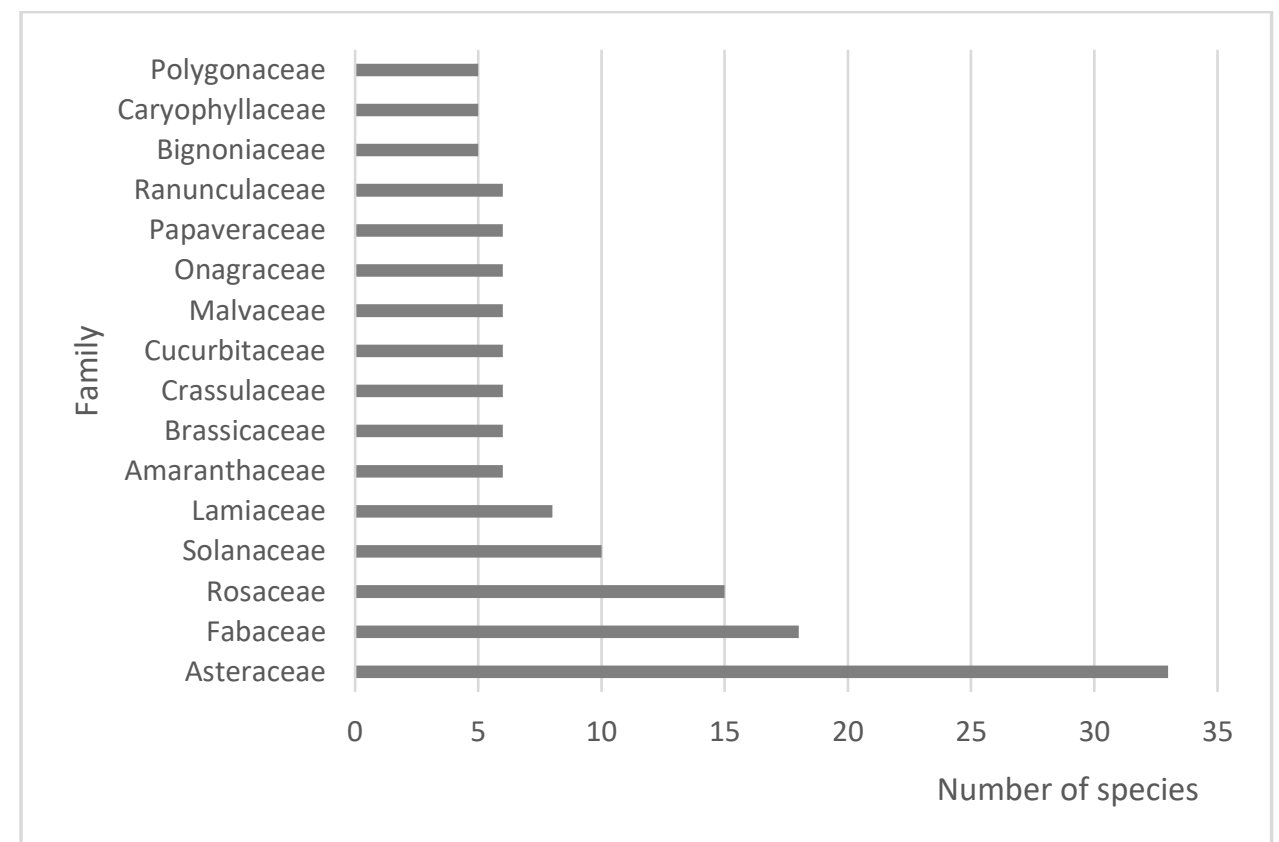

Fig. 1: The main families of the nNOP in Romania.

For Romania, the main contributers of non-native plants with ornamental value are America (100 taxa - 37.87\%), Asia (82 taxa - 31.06\%) and Mediterranean region (33 taxa 12.5\%) (Fig. 2, Table 1). The contribution of other regions such as Africa or Australia is very reduced (12 taxa - 4.54 taxa). Of hybrid origin there are 13 taxa (4.92\%) (Fig. 2).

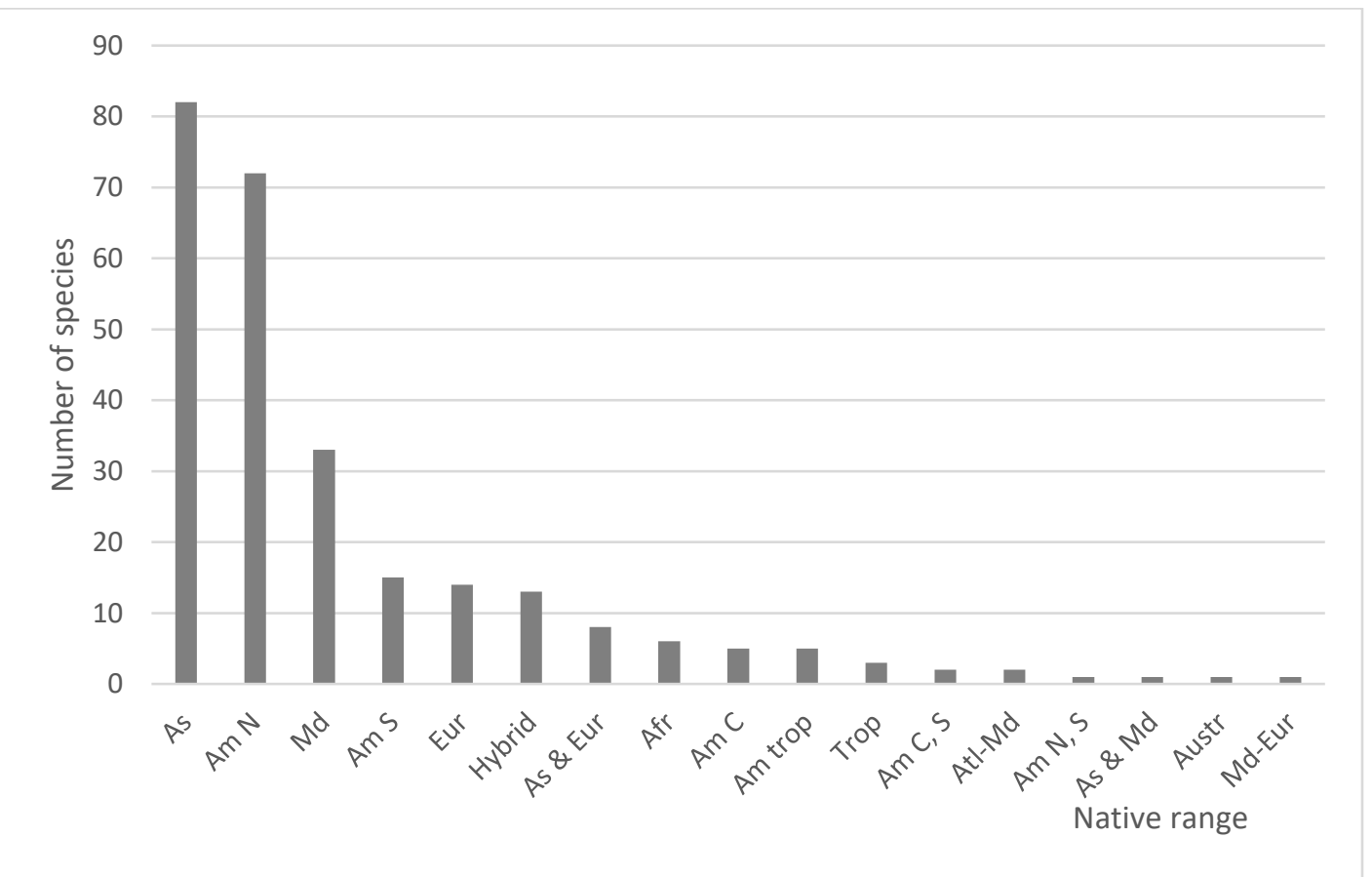

Fig. 2: Distribution of the nNOP in Romania according to the native range. Legend: Afr - Africa, Am NNorth America, Am S - South America, Am C - Central America, As - Asia, Atl - Atlantic, Austr Australia, Eur - Europe, Trop - Tropical, Md - Mediterranean. 
Regarding their life forms, the most nNOP are phanerophytes with 92 species $(34.84 \%)$ (Fig. 3, Table 1). They are followed by hemicryptophytes with 76 taxa $(28.78 \%)$, therophytes with 59 taxa (22.34\%), geophytes with 16 taxa (6.06\%), helohydrophytes with 8 taxa $(3.03 \%)$, chamaephytes and hemitherophytes with 4 taxa each (1.51\%), and others (5 taxa - about 2\%) (Fig. $3)$.

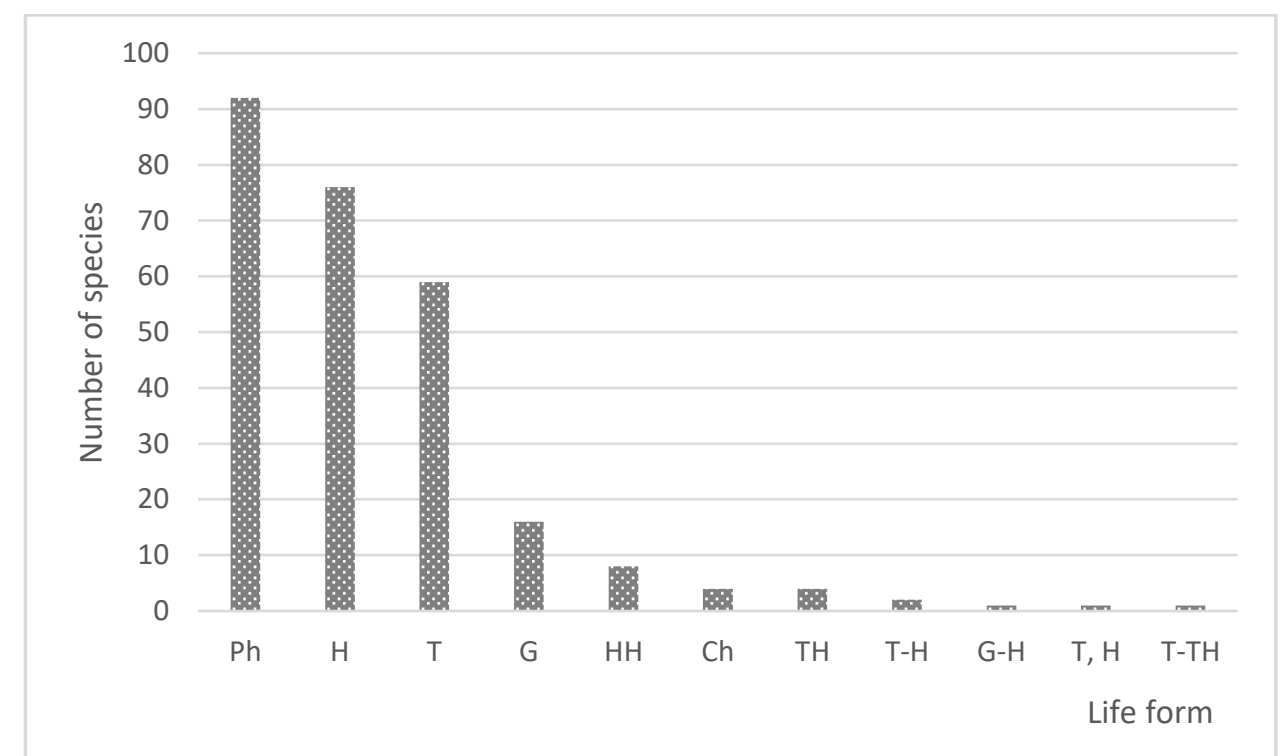

Fig. 3: Spectrum of life forms of the nNOP in Romania. Legend: $\mathrm{Ch}$ - Chamaephyte, $\mathrm{G}-\mathrm{Geophyte}, \mathrm{H}$ - Hemicryptophyte, HH - Helohydatophyte; Ph - Phanerophyte, T - Therophyte, TH Hemitherophyte.

A significant percent is represented by casual nNOP - 199 taxa (75.38\%) (Fig. 4, Table 1). Naturalised and invasive species together are less than a quarter (37 taxa $-14.02 \%$ naturalised and 28 taxa $-10.60 \%$ invasive) (Fig. 4). The ornamental species classified as invasive are: Acer negundo, Ailanthus altissima, Amaranthus hypochondriacus, Amorpha fruticosa, Asclepias syriaca, Bassia scoparia, Echinocystis lobata, Elaeagnus angustifolia, Fraxinus pennsylvanica, Helianthus tuberosus, Humulopsis scandens, Impatiens glandulifera, Lycium barbarum, Morus alba, Oenothera biennis, Oenothera glazioviana, Parthenocissus inserta, Prunus serotina, Reynoutria $\times$ bohemica, R. japonica, Robinia pseudoacacia, Rudbeckia laciniata, Sicyos angulatus, Sisyrinchium montanum, Solidago canadensis, S. gigantea, Symphiotrichum lanceolatum and S. salignum.

The most widespread species is Robinia pseudoacacia, already reported at national level from over 1000 localities. This is followed by a group of three species, all of them invasive, reported from 501-1000 localities: Bassia scoparia, Echinocystis lobata, Lycium barbarum. From 101 to 500 localities there are 20 non-native ornamental plant species, among these 16 being classified as invasive. Another group of 14 species is reported from 51 to 100 localities. This group includes four invasive species: Amaranthus hypochondriacus, Asclepias syriaca, Prunus serotina and Sisyrinchium montanum. 57 taxa are reported from a reduced number of localities, 11 to 50, three of them classified as invasive: Oenothera glazioviana, Sicyos angulatus, Symphiotrichum salignum. The largest group, with 108 taxa, includes species reported from two to ten localities. With only one single report there are 61 taxa (Fig. 5, Table 1). 


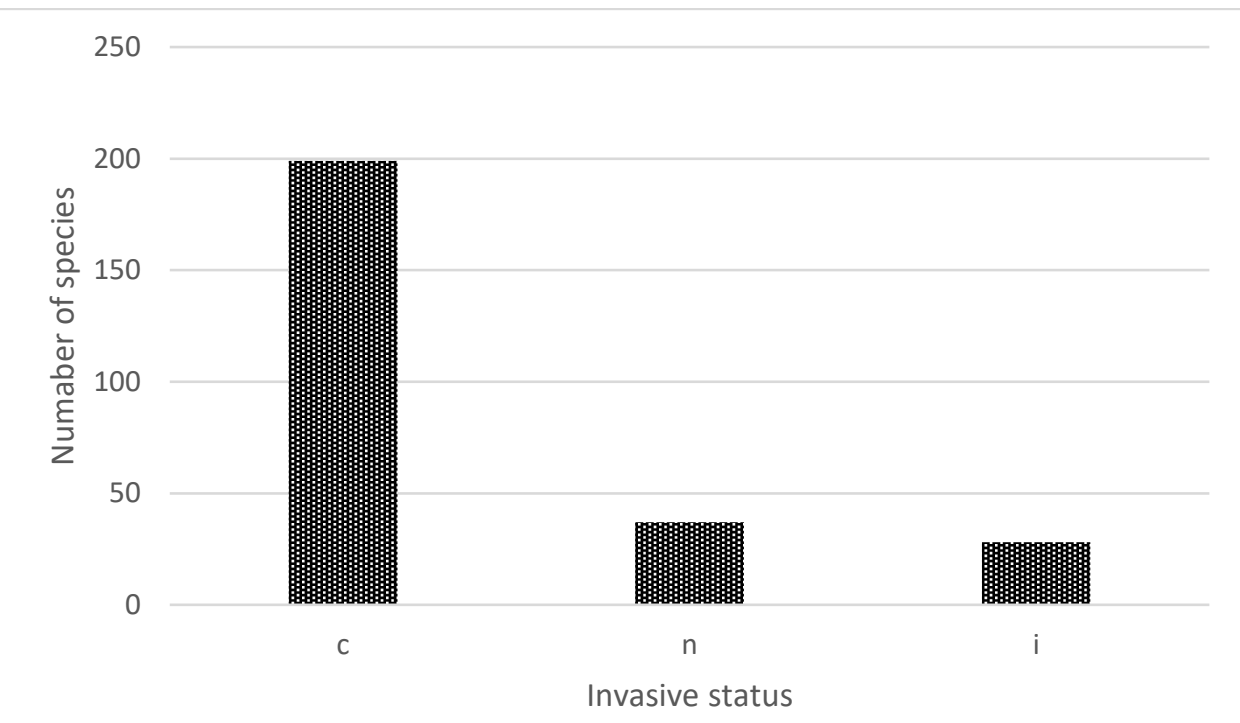

Fig. 4: Spectrum of invasiveness status of the nNOP in Romania. Legend: $c$ - casual, $n$ - naturalized, $i$ - invasive.

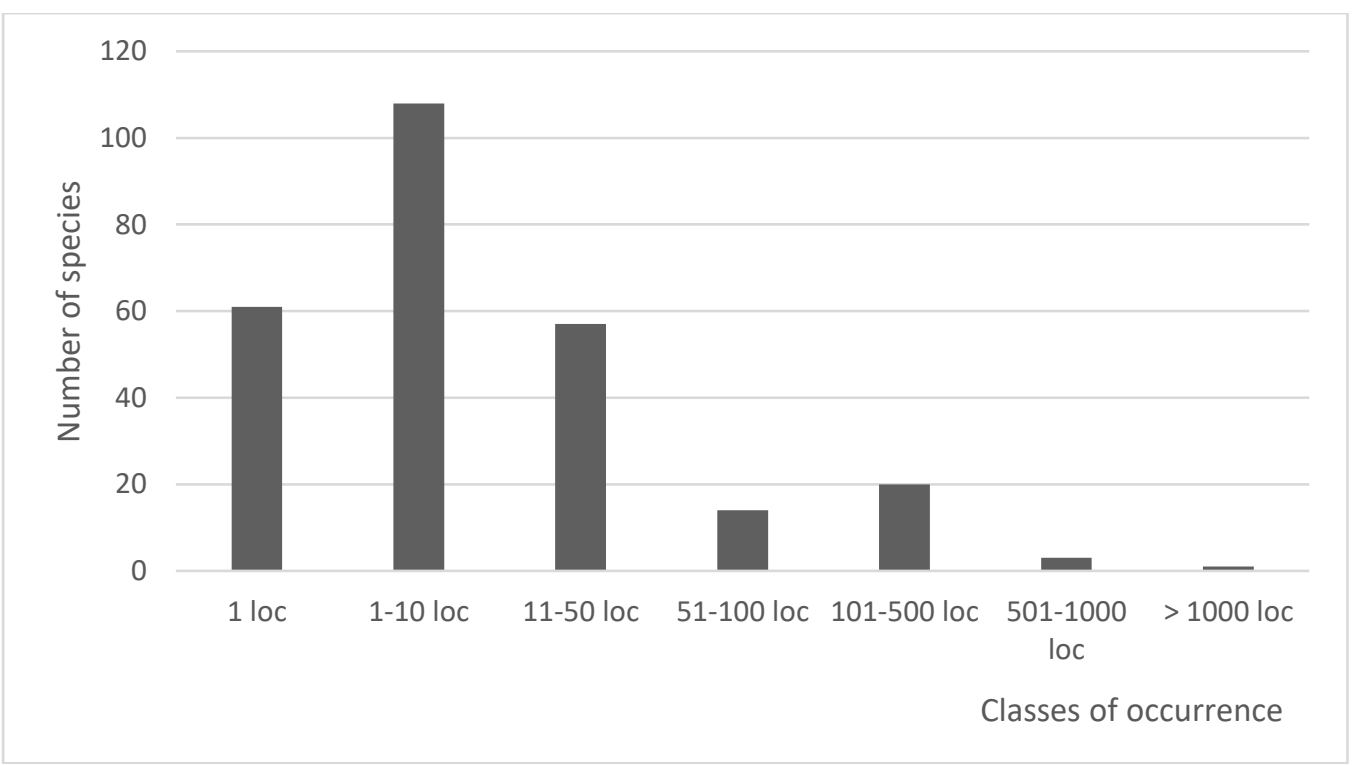

Fig. 5: Classes of occurrence of the nNOP in Romania.

More than half of the nNOP is reported only from artificial habitats (156 taxa $-59.09 \%)$, while a percent of $18.93 \%$ is represented by taxa reported from all types of habitats (Table 1).

First records of nNOP as sub-spontaneous date back to before 1800 (Calendula officinalis, Eranthis hyemalis, Nymphaea lotus and Symphiotrichum salignum). Before 1900 there have been reported 84 escapes among the $\mathrm{nNOP}(31.8 \%)$, while in the $20^{\text {th }}$ century there have been reported 102 escapes (38.63\%). In the last twenty years 78 (29.54\%) nNOP species have been reported as escaped from cultivation (Fig. 6). 


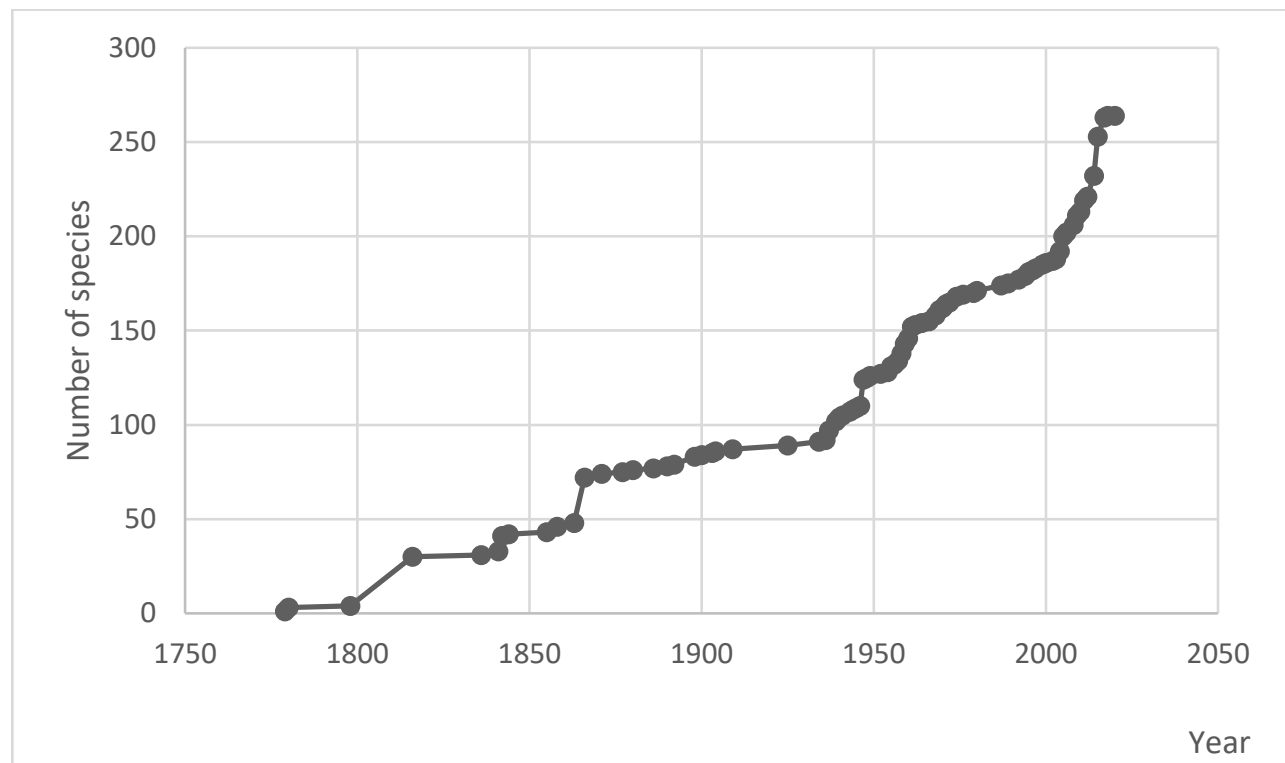

Fig. 6: Temporal trend of the nNOP in Romania in the last 240 years based on the year of first report of species as escaped from cultivation.

\section{Discussion}

The current list of nNOP in Romania includes about three times more taxa than the previous list published by Anastasiu et al. [1], fewer taxa than are indicated as ornamental by Sîrbu \& Oprea [46], and about a quarter of naturalised aliens in Europe reported as escapes of species cultivated for ornament and horticulture [37]. The previous list comprises 93 taxa [1], while Sîrbu \& Oprea [46] includes in their book 362 non-native plants with ornamental value. In Europe, escapes of species cultivated for ornament and horticulture account $58 \%$ of the total of 1983 naturalised aliens [37]. We did not include in the current list about 100 taxa not reported in the last 50 years, considering them to have vanished. Many have been reported as escaped from cultivation in botanical gardens, but did not survive over the time. We can mention some examples: Aquilegia $\times$ cultorum, Aquilegia chrysantha, A. coerulea, A. olimpica, A. skinneri reported in Bucharest Botanic Garden [13], or Kitaibelia vitifolia, Smilacina stellata, Solanum nodiflorum reported in Cluj-Napoca Botanic Garden [51]. On the other side, we included in the current list 78 species reported in the last 20 years. Among them are species reported as sub-spontaneous or naturalised in Bucharest Botanic Garden [34, 33] and in Jibou Botanic Garden as well [49, 35]. Two other species are missing from the current list due to taxonomic reasons. According to GBIF [19], Bassia sierversiana is a synonym of $B$. scoparia, and Fallopia aubertii is a synonym of $F$. baldshuanica. 23 taxa identified as nNOP for Romania are not included in EASIN Catalogue (accessed on 21-22 of November 2020). Mostly they are species recently reported as escaped (e.g. Alangium platanifolium, Aquilegia formosa, Aubrieta parviflora, Bulbine semibarbata, Catalpa fargesii, C. speciosa, Cenchrus alopecuroides, Cercis canadensis, C. griffithii, Cladrastis kentukea, Desmodium stenophyllum, Gazania rigens, Salvia desoleana, S. tiliifolia, Sassafras albidum).

Compared with the previous list [1], the number of families in the updated list is higher (47 families vs 80 families). The pattern of richness at the level of the most represented families is slightly changed, especially because of the higher number of taxa included in the current list, but also due to the different classification system used here [19]. In the current list, the top five families 
I.M. SÎRBU, P. ANASTASIU

are Asteraceae, Fabaceae, Rosaceae, Solanaceae and Lamiaceae. In the previous list the richest families were Asteraceae, Liliaceae, Fabaceae, Malvaceae, Ranunculaceae and Solanaceae with four taxa each [1]. Asteraceae and Fabaceae are also in the top five among the neophytes from Romania [3], as well as among the largest families of vascular plants of the world [10]. In fact, at global level, many species from Asteraceae family became popular as "garden ornamentals" due to the "diversity of plants, hardiness and reliability of flowering" [10]. The pattern of the families is similar to that of other countries such as Czech Republic [36] and Germany [31].

Regarding the pattern of the native range, the updated list is comparable with the previous one [1], and slightly different to the neophyte list for Romania, where American taxa are followed by Mediterranean and then Asian [3], and of the aliens list for Europe, where aliens of Asian origin comprise about $31 \%$, and those of American origin about 19\% [37].

The representation of life forms is slightly different to the previous list where the first rank belongs to hemicryptophytes with a percentage of $34.40 \%$, followed by phanerophytes with $25.80 \%$ [1], and totally opposite to the structure of life forms of neophytes from Romania, where therophytes represent the dominant category, followed by hemicryptophytes and phanerophytes [3]. Species reported as subspontaneous from botanic gardens contribute to the high number of phanerophytes included in this updated list. Thus, from Bucharest Botanic Garden the following woody species were reported as subspontaneous during the most recent period: Alangium platanifolium, Celastrus orbiculatus, Cercis canadensis, C. griffithii, Citrus trifoliata, Cladrastis kentukea, Desmodium stenophyllum and Sassafras albidum [34]. Other phanerophytes are identified as subspontaneous in Jibou Botanical Garden: Berberis julianae, Chaenomeles speciosa, Cotoneaster horizontalis, Euonymus fortune and Forsythia suspensa [49], while species of Catalpa were identified as subspontaneous in Văcăreşti Natural Park, Bucharest (Catalpa fargesii, C. ovata, C. speciosa) [8].

When we look to the group of invasive nNOP, with only one exception, all of them are included as aliens in the EASIN Catalogue (accessed on November 20-22, 2020) [18]. The missing species is Parthenocissus inserta, much more widespread in Romania than Parthenocissus quinquefolia (101 reports as opposed to 40 reports, according to Sîrbu \& Oprea [46]). Among the invasive nNOP, in the EASIN Catalogue 16 species are classified as being of high impact: Acer negundo, Ailanthus altissima, Asclepias syriaca, Amorpha fruticosa, Echinocystis lobata, Elaeagnus angustifolia, Helianthus tuberosus, Humulopsis scandens, Impatiens glandulifera, Morus alba, Prunus serotina, Robinia pseudoacacia, Reynoutria japonica, Sicyos angulatus, Solidago canadensis and S. gigantea. Reynoutria japonica has also been scored as of high socioeconomic impact in Europe [45]. In the category of naturalised nNOP there are also few species classified as of high impact in Europe, according to the EASIN Catalogue [18]. These are: Albizia julibrissin, Cabomba caroliniana, Gleditsia triacanthos, Lolium multiflorum, Reynoutria sachalinensis, Rhus typhina and Sagittaria latifolia. In fact, except Gleditsia triacanthos, none of these species is widely distributed in Romania. For example, Cambomba caroliniana, a species of EU concern, has been reported in two restricted sites in the north-west of the country [50], but its presence is not confirmed by recent data [7]. On the contrary, Gleditsia triacanthos is reported from about 180 localities [46], but according to our field observations the presence of juveniles is strictly correlated with the presence of cultivated adults. Moreover, this species is still much cultivated for afforestation in many parts of the country. nNOP reported as casual is the richest category when we discuss their invasiveness. Even when for some of them there are many reports 
(e.g. Calendula officinalis, Ipomoea purpurea and Tanacetum parthenium), they cannot establish persistent populations. According to EASIN Catalogue [18], 14 of the species identified as casual nNOP in Romania, are classified as species with high impact in Europe: Berberis aquifolium, $B$. thunbergii, Buddleja davidii, Celastrus orbiculatus, Erythranthe guttata, Lonicera japonica, Lupinus polyphyllus, Myriophyllum aquaticum, Paulownia tomentosa, Ricinus communis, Rosa rugosa, Symphyotrichum novi-belgii, Tamarix tetrandra and Tradescantia fluminensis. However, in Romania these species have a low occurrence (Table 1) and there have been no reports as yet of any negative impact correlated with their presence, even for those identified in natural habitats (Lonicera japonica, Symphiotrichum novii-belgii). For Buddleja davidii, a species scored as of high environmental impact in Europe [45], there are only four reports in Romania, from artificial and semi-natural habitats (Table 1).

While in Europe the most widely distributed alien taxa are Robinia pseudoacacia, Ailanthus altissima, Impatiens glandulifera, Reynoutria japonica and Rosa rugosa [37], in Romania the top five nNOP is occupied by Robinia pseudoacacia, Lycium barbarum, Echinocystis lobata, Bassia scoparia and Oenothera biennis (Table 1). This result is based on available data from literature, especially Sîrbu \& Oprea [46], but it could alter very soon, after the inventories obtained through the national project „POIM/178/4/1/120008 - Adequate management of invasive species in Romania, in accordance with EU Regulation 1143/2014 on the prevention and management of the introduction and spread of invasive alien species", implemented between 20182022, are published. The success of Robinia pseudoacacia could be explained by the multiple pathways of introduction (release in nature for erosion control, dune stabilization, afforestation or as a melliferous plant; escape for confinement: forestry, ornamental, melliferous [46], the long period of residence time (see Table 1), and the intensity of planting. According to Pyšek et al. [38], the probability of escapes of trees depends exclusively on planting intensity.

Data about the presence of nNOP in different types of habitat are very few and not satisfactory, thus this type of analysis require further investigation. However, a study on neophytes of wetlands in Romania indicates 29 non-native ornamental species from different types of habitats such as inland surface water habitats, riparian woodlands, riverine and lakeshore scrubs [2], which are known as vulnerable to invasion [27]. Non-native ornamental species such as Amorpha fruticosa, Acer negundo, Elaeagnus angustifolia and Robinia pseudoacacia are mentioned for their capacity to change the lakeshore habitats of Snagov lake [5]. Amorpha fruticosa and Robinia pseudoacacia have been scored as being of very high impact on the habitats in the "Iron Gates" Natural Park [20]. In habitats from Danube Delta Biosphere Reserve, Anastasiu et al. [6] reported as invasive some nNOP (Ailanthus altissima, Echinocystis lobata, Elaeagnus angustifolia, Fraxinus pennsylvanica, Lycium barbarum, Morus alba and Phytolacca americana), but pointed out that some species recorded as escaped from cultivation are known as invasive in other regions or countries: Asclepias syriaca, Helianthus tuberosus, Parthenocissus inserta, Rudbeckia laciniata and Solidago gigantea.

Analysis of temporal trends of nNOP escapes reveals that over the years, the reports about this category of plants increase constantly, except for the last twenty years, when we have noted an accelerated increase of reports regarding escaped nNOP (Fig. 6). Thus, in only 20 years the reports almost equal in number those for 100 years of recording.

In the current list of nNOP in Romania, six species are included in the updated list of invasive alien species of EU concern (17): Ailanthus altissima, Asclepias syriaca, Cabomba 
caroliniana, Humulopsis scandens, Impatiens glandulifera and Myriophyllum aquaticum. No management measures are implemented for their control and mitigation. Moreover, some species are still available for purchase from online stores (Humulopsis scandens, Cabomba caroliniana, and Asclepias syriaca).

\section{Conclusions}

As ornamental plants can be a significant source of introductions and spread into natural and semi-natural habitats, especially wetlands and sensitive zones such as natural protected areas, more attention should be given to this subject. Unequivocally, the problems posed by alien ornamental plants in Romania are similar to those faced in many other countries in Europe.

Thus, with regard to the benefits of ornamental plants, awareness of the most important stakeholders such as horticultural specialists, botanists, conservationists, and not least the general public, when importing, buying and using these plants needs to be as high as possible.

This paper is for the benefit of not only specialists but all categories of participants, adding the decisional factors that can play an important role in public awareness and improving legislation. The data have been compiled and analysed so as to offer supporting contributions of sustaining the need for development of proper tools for preventing, detecting, monitoring and managing the spread of nNOP.

When managing $\mathrm{nNOP}$ it is mandatory to take into consideration the obligations derived from European, international and national legislation regarding these taxa. Consequently, the collaboration between all stakeholders is also essential.

In the light of the above, several nNOP require special measures to control and mitigate their populations, as being species of European Union concern (Ailanthus altissima, Asclepias syriaca, Cabomba caroliniana, Humulopsis scandens, Impatiens glandulifera, Myriophyllum aquaticum). In addition, prevention of spread is without a doubt the preferred procedure rather than controlling the spread; therefore any nNOP reported as a casual deserves great attention.

The ornamental horticulture industry (including plant nurseries), along with botanic gardens, plays an important role in alien plant introductions, but also as a cause of their transport, propagation and spread. In order to mitigate the role of the ornamental plant industry in dispersing nNOP, we strongly recommend that the stakeholders involved adopt and implement the regulations of international and European codes of conduct and codes of practice regarding horticulture, ornamental and invasive alien plants, to implement voluntary and non-voluntary regulations for controlling the potentially invasive and invasive ornamental species (for example to label plants with information about the risk of escape and becoming invasive and proper care recommendations, to adopt good production practices and not dispose of plant waste outside places specially designed for the destruction of plant debris, ensure phytosanitary safety for products, etc.), to work with and invest in the scientific community that addresses nNOP, to use only sciencebased information, to work with volunteers and implement control initiative undertaken to remove ornamental alien plants that extend into natural habitats, to invest in continuously training and strengthening the personnel responsible, the gardening public and the buying public about the risk that nNOP may pose in order to prevent them become invasive, thus including stopping and avoiding the selling, buying and planting of the known IAS and make substitutes for invasives available, etc. 
These recommendations cannot be fulfilled without sustained actions of education and increased awareness among stakeholders, and a better knowledge of the characteristics of alien ornamental plants and the historical environmental changes that they have generated all over the world.

\section{REFERENCES}

1. Anastasiu, P., Negrean, G., Pascale, G., Liţescu, S., 2005, Plante ornamentale şi invazive în flora României. Lucrări ştiinţifice ale Universităţii de Știinţe Agricole şi Medicină Veterinară Ion Ionescu de la Brad, Iaşi, Seria Horticultură, 1(48): 619-624.

2. Anastasiu, P., Negrean, G., Başnou, C., Sârbu, C., Oprea, A., 2008, A preliminary study on the neophytes of wetlands in Romania. Pp. 180-190. In: Rabitsch, W., F. Essl, F., Klingenstein, F., (eds.), Biological Invasions - from Ecology to Conservation, Neobiota, 7: 180-190.

3. Anastasiu, P., Negrean, G., 2009, Neophytes in Romania. In: Rakosy, L., Momeu, L., (coord.), Neobiota in România, Presa Universitară Clujeană, Cluj-Napoca: 66-97.

4. Anastasiu, P., Negrean, G., Samoilă, C., Memedemin, D., Cogălniceanu, D., 2011, A comparative analysis of alien plant species along the Romanian Black Sea coastal area. The role of harbours, Journal of Coastal Conservation, 15: 595-606. DOI: 10.1007/s11852-011-0149-0.

5. Anastasiu, P., Lițescu, S., 2012, Preliminary study on the Flora of Snagov Lake Natural Reserve and its surroundings. Acta Horti Botanici Bucurestiensis, 39: 69-90.

6. Anastasiu, P., Negrean, G., Smarandache, D., Liţescu, S., Basnou, C., 2014, Neophytes in Protected Areas. Case Study: The Danube Delta Biosphere Reserve, Acta Horti Botanici Bucurestiensis, 41: 41-69.

7. Anastasiu, P., Preda, C., Bănăduc, D., Cogălniceanu, D., 2017, Invasive alien species of Union concern in Romania, Transylvanian Review of Systematical and Ecological Research "The Wetlands Diversity", 19(3): 93-106. DOI: 10.1515/trser-2017-0024.

8. Anastasiu, P., Camen-Comănescu, P., Nagodă, E., Lițescu, S., Negrean, G., 2017, Nature Reclaiming Its Territory in Urban Areas. Case Study: Văcăreşti Nature Park, Bucharest, Romania, Acta Horti Botanici Bucurestiensis, 44: 71-99. DOI: 10.1515/ahbb-2017-0005.

9. Bell, C.E., Wilen, C.A., Stanton, A.E., 2003, Invasive plants of horticultural origin, Hortscience, 38(1): 14-16. DOI: 10.21273/HORTSCI.38.1.14.

10. Christenhusz, M.J., Fay, M.F., Chase, M.W., 2017, Plants of the world: an illustrated encyclopedia of vascular plant, University of Chicago Press, Chicago.

11. Clement, E.J., Foster, M.C., 1994, Alien Plants of the British Isles: a provisional catalogue of vascular plants (excluding grasses), Botanical Society of the British Isles, London.

12. Dehnen-Schmutz, K., Holdenrieder, O., Jeger, M.J., Pautasso, M., 2010, Structural change in the international horticultural industry: some implications for plant health, Scientia Horticulturae, 125: 1-15. DOI: 10.1016/j.scienta.2010.02.017

13. Diaconescu, V., 1961, Sectorul "Plante Ornamentale" al Grădinii Botanice din București, Acta Botanici Horti Bucuretiensis, 1960: 71-118.

14. Drew, J., Anderson, N., Andow, D., 2010, Conundrums of a complex vector for invasive species control: a detailed examination of the horticultural industry, Biological Invasions, 12(8): 2837-2851. DOI: 10.1007/s10530-010-9689-8.

15. Dumitraşcu, M., Grigorescu, I., Năstase, M., Dragotă, C., Kucsicsa, G., 2010, The main environmental driving forces of the invasive plant species in the Romanian protected areas. In: Proceedings of BALWOIS Conference on Water Observation and Information Systems for Decision Support Ohrid, Macedonia (online). http://balwois.com/balwois/administration/full_paper/ffp-1600.pdf: 1-12.

16. Dumitraşcu, M., Kucsicsa, G., Grigorescu, I., Dragotă, C.S., Năstase, M., 2012, Invasive Terrestrial Plant Species in the Romanian Protected Areas. Case Study: Fallopia japonica in the Maramureş Mountains Natural Park, Forum geografic. Studii și cercetări de geografie și protecția mediului., 11(1):45-53. DOI: 10.5775/fg.2067-4635.2012.031.i. 
17. European Comission, 2019. Commission Implementing Regulation (EU) 2019/1262 of 25 July 2019 amending Implementing Regulation (EU) 2016/1141 to update the list of invasive alien species of Union concern. Official Journal of the European Union, L 199/1, 25 July 2019.

18. European Commission - Joint Research Centre - European Alien Species Information Network (EASIN) https://easin.jrc.ec.europa.eu/

19. GBIF (Global Biodiversity Information Facility). https://www.gbif.org/

20. Goia, I., Ciocănea, A.-M., Gavrilidis, A.-A., 2016, Geographic Origins of Invasive Alien Species in "Iron Gates" Natural Park (Banat, Romania), Transylvanian Review of Systematical and Ecological Research, 16(3): 115-130. DOI: 10.1515/trser-2015-0036.

21. Guo, W.-Y., van Kleunen, M., Pierce, S. Dawson, W., Essl, F., Kreft, H., Maurel, N., Pergl, J., Seebens, H., Weigelt, P., Pyšek, P., 2019, Domestic gardens play a dominant role in selecting alien species with adaptive strategies that facilitate naturalization, Global Ecology and Biogeography, 28(5): 628-639. DOI: $10.1111 /$ geb.12882.

22. Haeuser, E., Dawson, W., Thuiller, W., Dullinger, S., Block, S., Bossdorf, O., Carboni, M., Conti, L., Dullinger, I., Essl, F., Klonner, G., Moser, D., Münkemüller, T., Parepa M., Talluto, M.V., Kreft, H., Pergl, J., Pyšek, P., Weigelt, P., Winter, M., Hermy, M., van der Veken, S., Roquet, C., van Kleunen, M., 2018, European ornamental garden flora as an invasion debt under climate change, Journal of Applied Ecology, 55(5): 2386-2395. DOI: 10.1111/1365-2664.13197.

23. Halford, M., Heemers, L., Mathys, C., Vanderhoeven, S., Mahy, G., 2011, Socio-Economic Survey on Invasive Ornamental Plants in Belgium. Final report, February.

https://orbi. uliege. be/bitstream/2268/126498/1/Socio_economic_survey_EN. Pdf. Accesed 12 October 2020.

24. Heywood, V., Brunel, S., 2008, Code of conduct on horticulture and invasive alien plants. In: Council of Europe Convention on the Conservation of European Wildlife and Natural Habitats Standing Committee 28th meeting Strasbourg (Vol. 2427).

25. Hulme, P.E., Brundu, G., Carboni, M., Dehnen-Schmutz, K., Dullinger, S., Early, R., Essl, F., GonzalesMoreno, P., Groom, Q.J., Kueffer, C., Kühn, I., Maurel, N., Novoa, A., Pergl, J., Pyšek, P., Seebens, H., Tanner, R., Touza, J.M., van Kleunen, M., Verbrugge, L.N.H., 2018, Integrating invasive species policies across ornamental horticulture supply chains to prevent plant invasions, Journal of Applied Ecology, 55: 92-98. DOI: $10.1111 / 1365-2664.12953$.

26. Humair, F., Humair, L., Kuhn, F., Kueffer, C., 2015, E-commerce trade in invasive plants, Conservation Biology, 29: 1658-1665. DOI: 10.1111/cobi.12579.

27. Kowarik, I., 1999, Neophytes in Germany: quantitative overview, introduction and dispersal parthways, ecological consequences and open questions. In: Doyle, U. (ed.): Alien organisms in Germany. Proceedings of a Conference on Legal Regulations Concerning Alien organisms in comparison to Genetically Modified Organisms. Federal Environment Agency, Berlin, Texte 18/99: 12-36.

28. Kowarik, I., 2005, Urban ornamentals escaped from cultivation. In: Gressel, J. (ed.), Crop Ferality and Volunteerism: A Threat to Food Security in the Transgenic Era? CRC Press, Boca Raton.

29. Kühn, I., Klotz, S., 2002, Floristischer Status und gebietsfremde Arten, Schriftenreihe Vegetationskunde, 38: 47-56.

30. Maurel, N., Hanspach, J., Kühn, I., Pyšek, P., van Kleunen, M., 2016, Introduction bias affects relationships between the characteristics of ornamental alien plants and their naturalization success, Global Ecology and Biogeography, 25: 1500-1509. DOI: 10.1111/geb.12520.

31. Mayer, K., Haeuser, E., Dawson, W., Essl, F., Kreft, H., Pergl J., Pyšek, P., Weigelt, P., Winter, M., Lenzner, B., van Kleunen, M., 2017, Naturalization of ornamental plant species in public green spaces and private gardens, Biological invasions, 19(12): 3613-3627.

32. Memedemin, D., Anastasiu, P., Preda, C., Negrean, G., Cogălniceanu, D., 2016, Alien plant species turnover in Constanţa Harbor (Romania) in the last decade, Acta Horti Botanici Bucurestiensis, 43: 5-18. DOI: 10.1515/ahbb-2016-0001

33. Nagodă, E., 2015, Research on alien plants from Bucharest and surroundings, PhD thesis, University of Bucharest, Romania.

34. Nagodă, E., Comănescu, P., Anastasiu, P., 2014, „Dimitrie Brandza” Botanic Garden, Potential Centre for the Dispersal of Invasive Plants? Acta Horti Botanici Bucurestiensis, 41: 13-40. DOI: 10.2478/ahbb-2014-0002. 
35. Negrean G., Karácsonyi, C., Szatmari P.M., 2017, Patrimoniu natural al Sălajului. Vol. Flora, microbiota şi vegetaţia, Satu Mare: Edit. "Someşul".

36. Pergl, J., Sádlo, J., Petřik, P., Danihelka, J., Chrtek, Jr. J., Hejda, M., Moravcová, L., Perglová, I., Štajerová, K., Pyšek, P., 2016, Dark side of the fence: ornamental plants as a sourse of wild-growing flora in the Czech Republic, Preslia, 88: 163-184.

37. Pyšek, P., Lambdon, P.W., Arianoutsou, M., Kühn, I., Pino, J., Winter, M., 2009, Alien Vascular Plants in Europe. In: Hulme, P., Netwig, W., Pyšek, P., Vilà, M. (eds.), The handbook of alien species in Europe. Springer Publisher, Dordrecht-Heidelberg: 43-61.

38. Pyšek, P., Křivánek, M., Jarošík, V., 2009, Planting intensity, residence time, and species traits determine invasion success of alien woody species, Ecology, 90(10): 2734-2744. DOI: 10.1890/08-0857.1.

39. Pyšek, P., Jarošík, V., Hulme, P.E., Pergl, J., Hejda, M., Schaffner, U., Vilà, M., 2012, A global assessment of invasive plant impacts on resident species, communities and ecosystems: the interaction of impact measures, invading species' traits and environment, Global Change Biology, 18(5): 1725-1737. DOI: 10.1111/j.13652486.2011.02636.x.

40. Pyšek, P., Richardson, D.M., Rejmanek, M., Webster, G.L., Williamson, M., Kirschner, J., 2004, Alien plants in checklists and floras: towards better communication between taxonomists and ecologists, Taxon, 51(1): 131143. DOI: $10.2307 / 4135498$

41. Pyšek, P., Richardson, D.M., 2007, Traits Associated with Invasiveness in Alien Plants: Where do We Stand? In: Nentwig, W., (ed.), Biological invasions. Springer, Berlin.

42. Raunkiaer, C., 1934, The life forms of plants and statistical plant geography, Clarendon Press, Oxford

43. Reichard, S.H., White, P., 2001, Horticulture as a pathway of invasive plant introductions in the United States: most invasive plants have been introduced for horticultural use by nurseries, botanical gardens, and individuals, BioScience, 51(2): 103-113. DOI: 10.1641/0006-3568(2001)051[0103:HAAPOI]2.0.CO;2.

44. Richardson, D.M., Pyšek, P., Rejmánek, M., Barbour, M.G., Panetta, F.D., West, C.J., 2000, Naturalization and invasion of alien plants: concepts and definitions, Diversity and distributions, 6(2): 93-107. DOI: 10.1046/j.1472-4642.2000.00083.x

45. Rumlerová, Z., Vilà, M., Pergl, J., Nentwig, W., Pyšek, P., 2016, Scoring environmental and socioeconomic impacts of alien plants invasive in Europe, Biological invasions, 18(12): 3697-3711. DOI: 10.1007/s10530016-1259-2.

46. Sîrbu, C., Oprea, A., 2011, Plante adventive în flora României, Editura "Ion Ionescu de la Brad", Iași.

47. Sîrbu, C., 2007, Considerations regarding the alien plants from Moldavian flora (Romania), deliberately introduced by man, Journal of Plant Development, 14: 41-50.

48. Sîrbu, C., Oprea, A., Pavol, E., jun., Ferus, P., 2011, New contribution to the study of alien Flora in Romania, Journal of Plant Development, 18: 121-134.

49. Szatmari, P.M., Căprar, M., 2015, Research on spontaneous and subspontaneous flora of Botanical Garden 'Vasile Fati' Jibou, Journal of Horticulture, Forestry and Biotechnology, 9(2): 176 - 189.

50. Țopa, E., 1955, Familia Nymphaeaceae. In: Săvulescu, T. (ed.), Flora României. Vol. 3. București: Editura Academiei Române, Bucureşti: 34-49.

51. Țopa, E., 1947, Contribuțiuni noui la cunoașterea ergasiophygophytelor din Cluj, Buletinul Grădinii Botanice şi al Muzeului Botanic de la Universitatea din Cluj, 27(3-4): 181-188.

52. van Kleunen, M., Essl, F., Pergl, J, Brundu, G., Carboni, M., Dullinger, S., Early, R., González-Moreno, P., Groom, Q.J., Hulme, P.E., Kueffer, C., Kühn, I., Máguas, C., Maurel, N., Novoa, A., Parepa, M., Pyšek, P., Seebens, H., Tanner, R., Touza, J., Verbrugge, L., Weber, E., Dawson, W., Kreft, H., Weigelt, P., Winter, M., Klonner, G., Talluto, M.V., Dehnen-Schmutz, K., 2018, The changing role of ornamental horticulture in alien plant invasions, Biological Reviews, 93(3): 1421-1437. DOI: 10.1111/brv.12402.

53. van Kleunen, M., Weber, E., Fischer, M., 2010, A metaanalysis of trait differences between invasive and noninvasive plant species, Ecology Letters, 13: 235-245. DOI: 10.1111/j.1461-0248.2009.01418.x. 


\section{LISTA ACTUALIZATĂ A PLANTELOR ORNAMENTALE EXOTICE DIN ROMÂNIA}

\section{(Rezumat)}

Prima listă a plantelor ornamentale exotice scăpate din cultură, naturalizate sau invasive a fost publicată în urmă cu 15 ani. De atunci, s-au înregistrat noi raportări de specii ornamentale pentru România, în timp ce altele şi-au schimbat statutul. Cum horticultura ornamentală este în întreaga lume una dintre cele mai importante căi de pătrundere pentru plantele exotice, am evaluat situaţia acestora la nivel naţional şi am actualizat lista publicată anterior cu scopul de a contribui la dezvoltarea instrumentelor de prevenire, detectare, monitorizare şi cartare a distribuţiei plantelor ornamentale exotice. Lista actualizată include 264 taxoni. Cei mai mulţi sunt doar ocazional întâlniţi ca subspontani (199 taxoni), 37 sunt naturalizaţi şi 28 sunt invazivi. Au fost analizate următoarele caracteristici: familia, distribuţia nativă, forma biologică, prezenţa în ţară (număr de localităţi) şi tendinţele în timp. Flora ornamentală exotică din România este dominată de specii americane şi asiatice, fanerofite, hemicriptofite şi terofite. Cea mai răspândită este Robinia pseudoacacia, folosită nu doar în scopuri ornamentale ci şi pentru valoarea sa economică. Cel mai mulţi taxoni (108) sunt răspândiţi în două până la 10 localităţi. În ultimii 20 de ani au fost raportate 78 de specii exotice ornamentale noi pentru România. Şase taxoni sunt îngrijorători pentru Uniunea Europeană (Ailanthus altissima, Asclepias syriaca, Cabomba caroliniana, Humulopsis scandens, Impatiens glandulifera, Myriophyllum aquaticum) şi necesită măsuri de control şi reducere a populaţiilor lor. 